\title{
No X-rays from WASP-18
}

\section{Implications for its age, activity, and the influence of its massive hot Jupiter ${ }^{\star}$}

\author{
Ignazio Pillitteri ${ }^{1,2}$, Scott J. Wolk ${ }^{2}$, Salvatore Sciortino ${ }^{1}$, and Victoria Antoci ${ }^{3}$ \\ 1 Osservatorio Astronomico di Palermo, Piazza del Parlamento 1, 90134 Palermo, Italy \\ e-mail: pilli@astropa.inaf.it \\ 2 SAO-Harvard Center for Astrophysics, 60 Garden St, Cambridge MA 02138, USA \\ 3 Stellar Astrophysics Centre, Department of Physics and Astronomy, Aarhus University, Ny Munkegade 120, 8000 Aarhus C, \\ Denmark
}

Received 5 February 2014 / Accepted 10 June 2014

\begin{abstract}
About $20 \%$ of the $>1000$ known exoplanets are Jupiter analogs orbiting very close to their parent stars. It is still under debate to what detectable level such hot Jupiters possibly affect the activity of the host stars through tidal or magnetic star-planet interaction. In this paper we report on an $87 \mathrm{ks}$ Chandra observation of the hot Jupiter hosting star WASP-18. This system is composed of an F6 type star and a hot Jupiter of mass $10.4 M_{\text {Jup }}$ orbiting in less than $20 \mathrm{hr}$ around the parent star. On the basis of an isochrone fitting, WASP-18 is thought to be $600 \mathrm{Myr}$ old and within the range of uncertainty of $0.5-2 \mathrm{Gyr}$. The star is not detected in X-rays down to a luminosity limit of $4 \times 10^{26} \mathrm{erg} / \mathrm{s}$, which is more than two orders of magnitude lower than expected for a star of this age and mass. This value proves an unusual lack of activity for a star with an estimated age around $600 \mathrm{Myr}$. We argue that the massive planet can play a crucial role in disrupting the stellar magnetic dynamo created within its thin convective layers.

Other additional $212 \mathrm{X}$-ray sources are detected in the Chandra image. We list them and briefly discuss their nature.
\end{abstract}

Key words. stars: activity - stars: coronae - stars: individual: WASP-18

\section{Introduction}

About $20 \%$ of the $>1000$ extra solar planets discovered to date are hot Jupiters, meaning massive planets orbiting at a few stellar radii from the parent stars. Models predict that hot Jupiters could affect the activity of their host stars through either tidal or magneto-hydrodynamical interaction (e.g. Cuntz et al. 2000 and Ip et al. 2004). Both effects strongly scale with the separation $d$ between the two bodies (Saar et al. 2004). Observational evidence of star-planet interaction (SPI) was first reported by Shkolnik et al. (2003), who discovered variability in the chromospheric activity indicators, the $\mathrm{H}$ and $\mathrm{K}$ lines of $\mathrm{Ca}$ II of HD 179949, phased with the planetary motion. Subsequently, Fares et al. (2010) have reconstructed the magnetic field of HD 179949 and confirm periodic variations of chromospheric activity indicators ( $\mathrm{H} \alpha$ and $\mathrm{Ca}$ II lines) synchronized with the beat period of the planet-star system.

In the X-ray band, Kashyap et al. (2008) show that stars with hot Jupiters are statistically brighter by up to a factor four than stars with distant planets. Krejčová \& Budaj (2012) support the findings of Kashyap et al. (2008) by means of a survey of Ca II H and $\mathrm{K}$ lines on planet hosting stars that traces a relationship betweeen stellar activity and planet-star separation, with closer hot Jupiters orbiting more active stars. However, Poppenhaeger et al. (2010) and Poppenhaeger \& Schmitt (2011) argue that that the above results are biased by selection effects, and SPI is not a

* Full Table 2 is available in electronic form at http: //www . aanda.org common phenomenon, but instead SPI only manifests itself in peculiar cases.

One of the clearest cases of detection of SPI is HD 189733. We have observed this system three times with XMM-Newton and found strong evidence of SPI at work in X-rays (Pillitteri et al. 2010, 2011, 2014). The first evidence comes from the overall activity of the host star. The very low level of X-ray activity of the M type companion, HD $189733 \mathrm{~B}$, puts a strong constraint on the age of the system at $\geq 2$ Gyr (Pillitteri et al. 2010; Poppenhaeger et al. 2013). Supported also by the 2011 and 2012 XMM-Newton observations, the old age of HD 189733B is reinforced by the fact that it does not show flaring variability on a time scale of a few hours and within the XMM-Newton exposures (30-50 ks), as in young or active M-type stars. The old age of the secondary is inconsistent with the young age of the system as derived from stellar activity of HD 189733A, which is about 600 Myr (Melo et al. 2006). Schröter et al. (2011) report a similar result on CoRoT-2A. Based on Chandra observations of a planetary transit, they find that the primary is X-ray bright with a luminosity $\sim 1.9 \times 10^{29} \mathrm{erg} \mathrm{s}^{-1}$, indicating an age $<300 \mathrm{Myr}$, while a potential stellar companion of CoRoT-2A is undetected down to a limit of $L_{\mathrm{X}} \sim 9 \times 10^{26} \mathrm{ergs} \mathrm{s}^{-1}$, implying a much older age.

In main sequence stars, coronal activity is tightly linked with the internal structure of the stars, because of the link between convective zone, dynamo action, and magnetic field emergence. Stellar activity is a handle for understanding the depth of the convective zone and the efficiency of the dynamo. In stars with intermediate masses, approximately from late A-type stars and 
Table 1. Main properties of the WASP-18 system.

\begin{tabular}{|c|c|c|c|c|c|c|c|}
\hline $\begin{array}{l}\text { Name } \\
\text { WASP-18 }\end{array}$ & $\begin{array}{c}\text { Type } \\
\text { F6IV-V }\end{array}$ & $\begin{array}{c}\text { Mass } \\
1.28 \pm 0.09 M_{\odot}\end{array}$ & $\begin{array}{c}\text { Radius } \\
1.29 \pm 0.16 R_{\odot}\end{array}$ & $\begin{array}{c}\text { Distance } \\
100 \pm 10 \mathrm{pc}\end{array}$ & $\begin{array}{c}T_{\text {eff }} \\
6400 \pm 75 \mathrm{~K}\end{array}$ & $\begin{array}{c}V \\
9.3 \mathrm{mag}\end{array}$ & $\begin{array}{c}\text { Age } \\
0.63(0.5-2 \mathrm{Gyr})\end{array}$ \\
\hline Name & Type & Mass & Radius & Period & Semi-major axis & Note & \\
\hline WASP-18b & hot Jupiter & $10.4 \pm 0.4 M_{\text {Jup }}$ & $1.165 \pm 0.077 R_{\text {Jup }}$ & $0.9414518 \pm 4 \times 10^{-7} \mathrm{~d}$ & $0.0205 \pm 0.0004 \mathrm{AU}$ & Transiting & \\
\hline
\end{tabular}

Notes. Photospheric data from Doyle et al. (2013), other data from the catalog at exoplanet.eu. The range of age is from Southworth et al. (2009).

moving toward earlier types, the thin convective layer disappears, and so do magnetic dynamo and coronal emission. The precise onset of the convection is a function of the mass, the chemical composition, which affects the opacity of the inner layers of the star, and the stellar evolutionary stage. Mid F-type stars like our target, WASP-18, should possess a thin convective layer that can still generate an $\alpha-\omega$ dynamo similar to the solar one and produce a X-ray bright corona. In fact, mid and late F stars are X-ray bright in young clusters like the Hyades (600 Myr) at a level of $L_{X}>10^{28.5} \mathrm{erg} / \mathrm{s}$. In the framework of the relationship between age, rotation, convective zone depth, dynamo, and coronal activity, the emission of X-rays in this range of masses is a probe of the dynamo efficiency from thin convective layer coupled with the rotation of the star. In late F-type stars with hot Jupiters at the age of Hyades, an enhancement of the X-ray activity should then be expected.

With the intent of exploring SPI as a function of the starplanet separation in a star with shallow convective zone, we observed the system of WASP-18 with Chandra for a whole orbital period of its planet. WASP-18 (HD 10069, 2MASS J013725034540404) is a F6 star at $\sim 100 \mathrm{pc}$ from the Sun that harbors a very close-in hot Jupiter, with the planet orbiting in only $19.4 \mathrm{~h}$ (Hellier et al. 2009). The main characteristics of this system are given in Table 1 and are obtained from a recent spectroscopic study by Doyle et al. (2013). Numerous optical spectroscopic observations allowed quite precise estimates of the effective temperature, gravity, distance, chemical abundances and mass of WASP-18 (Doyle et al. 2013).

WASP-18b has a mass of about $10.43 M_{\text {jup }}$ and a density $\rho=6.6 \rho_{\text {Jup }}$. Owing to a star-planet separation of only $3.48 R_{*}$ $(0.02047 \mathrm{AU})$, the planet is experiencing strong irradiation that heats and bloats its atmosphere and fills its Roche lobe. Southworth et al. (2009) estimate an equilibrium temperature of about $2400 \mathrm{~K}$. The close separation suggests that the planet is on the verge of the final spiralling phase toward the parent star and this gives an opportunity to observe the final phases of a planet before disruption (Brown et al. 2011).

While WASP-18 was known to have low activity based on the $\log R_{\mathrm{HK}}^{\prime}$ indicator, the remarkably close separation between planet and star demands further studies of this system to explore the effects of SPI, both of tidal and magnetical origins. A star with an age of $600 \mathrm{Myr}$ is expected to have a level of X-ray luminosity typical of that of Hyades $\left(L_{X} \sim 10^{28.5}-10^{29.5} \mathrm{erg} / \mathrm{s}\right.$, Stern et al. 1995; Randich \& Schmitt 1995). Despite this range of presumed X-ray luminosity, WASP-18 was undetected in a $50 \mathrm{ks}$ stacked Swift exposure (Miller et al. 2012) at a limit of $\log L_{X, \lim }=27.5$, and in the ROSAT All Sky Survey (which has a typical sensitivity of $\log f_{\mathrm{X}} \geq 10^{-12} \mathrm{erg} \mathrm{s}^{-1} \mathrm{~cm}^{-2}$ ).

In this paper, we report the absence of detected X-ray emission from WASP-18 in a $87 \mathrm{ks}$ deep Chandra exposure and its implications for the models of star-planet interaction and the evolutionary stage of this system. The structure of the paper is as follows. Section 2 describes the information on the age of WASP-18, Sect. 3 describes the observations and data analysis, and Sect. 4 reports our results. Finally, in Sects. 5 and 6 we discuss the results and draw our conclusions.

\section{Age of WASP-18}

With respect to our investigations the age of WASP-18 is a critical parameter, hence in this section. we discuss it in details. Estimating the age of a star is a difficult task, and best applied to a statistical sample like stars belonging to open clusters. Methods for dating the age of stars are semi- or fully empirical and rely on gyrochronology, rotation and activity tracers, or on isochrone fitting or asteroseismology, the last used especially for solar-like oscillators and red giants (Soderblom 2010). It is observed that stars during the main sequence phase lose angular momentum through magnetized stellar winds, so that for single stars it is possible to estimate their age from their rotation (Skumanich 1972).

Historically, activity tracers have been the $\mathrm{Ca}$ II $\mathrm{H}$ and $\mathrm{K}$ lines, Mg II H and K lines and $\mathrm{H} \alpha$ line (Wilson 1966; Vaughan \& Preston 1980; Baliunas et al. 1995). All of them are sensitive to the chromospheric contribution to the line that is related to the overall stellar activity. Again, the connection between activity and rotation and between rotation and age makes measurement of these lines an empirical method for estimating the stellar age. However, in the cases of stars with hot Jupiters, the rotation and the activity tracers can be affected by the interaction with the planet, thus biasing the estimate of the age of the host star. Pillitteri et al. (2010, 2011, 2014); Poppenhaeger et al. (2013); Poppenhaeger \& Wolk (2014) and Schröter et al. (2011) have found that the hot Jupiter hosting stars HD 189733 and CoRoT-2A have most likely been spun up by their close in planets, and thus their activity and rotation have been enhanced, thereby mimicking the behavior of younger stars. In HD 189733, activity tracers like X-ray luminosity would assign an age in the range 0.6-1.1 Gyr (Melo et al. 2006; Sanz-Forcada et al. 2011), while the stellar companion is much older. On the same star, Torres et al. (2008) used fitting to $Y^{2}$ isochrones (Demarque et al. 2004; Yi et al. 2001) to estimate an age of $\tau=6.8_{-4.4}^{+5.2} \mathrm{Gyr}$.

The low chromospheric activity of WASP-18 would assign to it an age similar to that of the Sun or older. On the basis of isochrone fitting, the age of WASP-18 has been estimated by Hellier et al. (2009) and Southworth et al. (2009) to be similar to that of the Hyades but this estimate has a large range of uncertainty. Southworth et al. (2009) studied in details the stellar parameters of WASP-18 employing several models of stellar evolution: models from Claret and collaborators (Claret 2004, 2005, 2006, 2007), $Y^{2}$ models (Demarque et al. 2004; Yi et al. 2001), and Cambridge models (Eldridge \& Tout 2004; Pols et al. 1998). The parameters from various models agree well with each other (less agreement is found for the results from Cambridge models), but overall the age of WASP-18 is found in the range from 0.5 to at most 2 Gyr. We assume this range of age for WASP-18 and in Sect. 4 we compare these values against the evidences of an older stellar age. For a star in 
Table 2. List of X-ray sources detected in the ACIS image.

\begin{tabular}{lrrrrrrrrrr}
\hline \hline Num. & $\begin{array}{r}\text { RA (J2000) } \\
\text { deg }\end{array}$ & $\begin{array}{r}\text { Dec } \begin{array}{r}\text { (J2000) } \\
\text { deg }\end{array} \\
\text { arcsec }\end{array}$ & $\begin{array}{r}\text { Pos. err } \\
\text { arcmin }\end{array}$ & $\begin{array}{r}\text { Off-axis } \\
\text { arcmificance } \\
\sigma_{\text {bkg }}\end{array}$ & $\begin{array}{r}\text { Counts } \\
\text { cts }\end{array}$ & $\begin{array}{r}\text { Cts err. } \\
\text { Rate }\end{array}$ & $\begin{array}{r}\text { Rate err. } \\
\text { ct ks }^{-1}\end{array}$ & $\begin{array}{r}\text { Exp. time } \\
\text { ks }\end{array}$ \\
\hline 1 & 24.23177 & -45.54562 & 8.4 & 9.78 & 4.45 & 69.51 & 21.66 & 1.99 & 0.62 & 34.93 \\
2 & 24.24039 & -45.58173 & 2.5 & 7.77 & 4.13 & 18.18 & 7.29 & 0.296 & 0.119 & 61.37 \\
3 & 24.24631 & -45.52741 & 3.3 & 10.46 & 4.38 & 24.7 & 9.64 & 0.79 & 0.308 & 31.25 \\
4 & 24.25307 & -45.62677 & 1 & 5.41 & 9.44 & 30.27 & 9.06 & 0.399 & 0.119 & 75.95 \\
5 & 24.26107 & -45.64003 & 0.8 & 4.66 & 4.77 & 7.54 & 4.5 & 0.123 & 0.073 & 61.51 \\
6 & 24.26128 & -45.59062 & 1.9 & 6.83 & 4.2 & 13.33 & 5.74 & 0.183 & 0.079 & 72.66 \\
7 & 24.26154 & -45.59641 & 2.5 & 6.54 & 4.39 & 18.43 & 7.22 & 0.249 & 0.097 & 74.09 \\
8 & 24.26997 & -45.53962 & 2.2 & 9.4 & 12.45 & 76.95 & 11.65 & 1.359 & 0.206 & 56.61 \\
9 & 24.27015 & -45.62587 & 1.4 & 4.93 & 8.56 & 32.49 & 9.56 & 0.409 & 0.12 & 79.52 \\
10 & 24.27129 & -45.58701 & 2 & 6.79 & 7.52 & 35.92 & 10.77 & 0.487 & 0.146 & 73.81 \\
\hline
\end{tabular}

Notes. Only ten rows are shown here. The full table is available online.

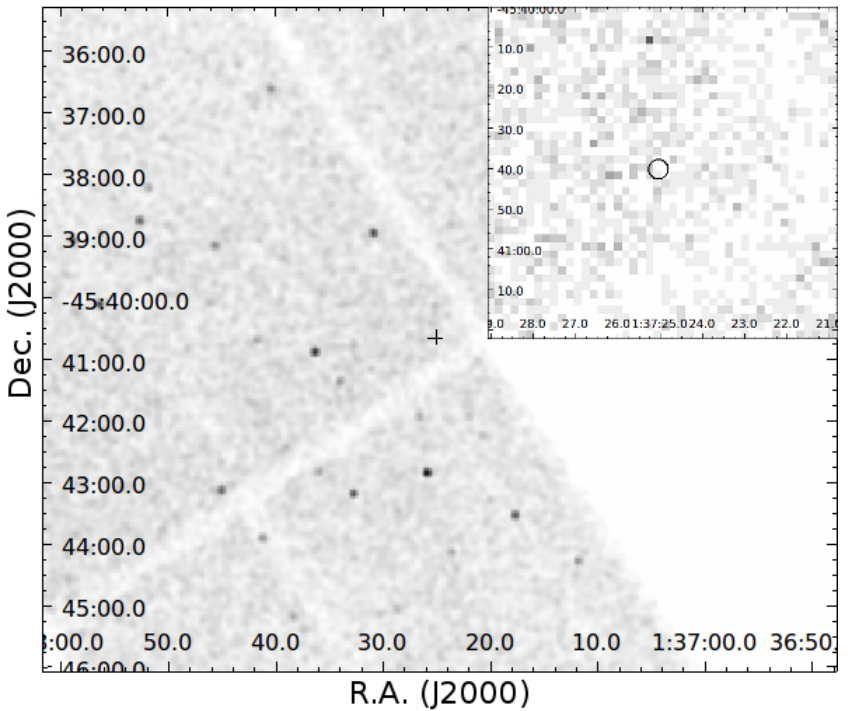

Fig. 1. Chandra-ACIS image toward WASP-18. The cross marks the position reported in the SIMBAD database. The inset image in the top right corner shows the region around the star, with the circle marking the position of WASP-18.

the 0.5-2 Gyr age time interval, corresponding to an age between that of the Hyades and of stars in NGC 752 open cluster, the X-ray luminosity of late F stars should be approximately in the range $28.1<\log L_{\mathrm{X}}<29.5$ (Pallavicini et al. 1981; Stern et al. 1995; Randich \& Schmitt 1995; Giardino et al. 2007).

\section{Observation and data analysis}

We observed WASP-18 $\left(\alpha=1^{\mathrm{h}} 37^{\mathrm{m}} 24.2^{\mathrm{s}}, \delta=-45^{\mathrm{d}} 40^{\mathrm{m}} 40.3^{\mathrm{s}}\right.$, J2000) using Chandra with a continuous $\sim 87$ ks long observation with ACIS camera. ACIS CCDs number 1, 2, 3, 6, 7 were used. WASP-18 falls in the CCD nr. 3 (see Fig. 1). The star is not visible in the X-ray image and is not detected at a significance threshold of $4 \sigma$ of local background, after applying a wavelet detection algorithm (Damiani et al. 1997b,a, 2003). The significance threshold of $4 \sigma$ is the value usually adopted to statistically have at most one spurious source per field. We also ran the detection algorithm at a significance threshold of $3 \sigma$, but still no sources are found within $5^{\prime \prime}$ from the nominal position of the star. We tested the hypothesis that WASP-18 could be heavily embedded in the material stripped from the outer atmosphere of the planetary companion. In this case the gas absorption should attenuate the soft part of the spectrum $(k T<1 \mathrm{keV})$, leaving any hard component spectrum still detectable. For this purpose we applied the same source detection procedure on the image in the $1.5-5.0 \mathrm{keV}$ band without successful detection of any source at the position of WASP-18. Hence we exclude that WASP-18 is shrouded in X-rays by a dense gas layer from its hot Jupiter. At this point, we calculated an upper limit to the rate in $0.3-8.0 \mathrm{keV}$ consistent with the $3 \sigma$ threshold, and this value is $3.8 \times 10^{-5}$ counts $\mathrm{s}^{-1}$.

Table 2 reports the positions, offaxis, significance, counts, count rates, and exposure times of 212 detected sources detected above the $4 \sigma$ threshold. We cross-correlated the positions of the detected sources against NED, 2MASS and Simbad catalogs in order to identify optical and IR counterparts. Table 4 reports the list of matches with notes about their nature. For the brightest sources, we also extracted the spectra, and performed a best fit, adopting either an absorbed thermal model or an absorbed power law with XSPEC software ver. 12.8. Table 5 lists the best fit parameters.

We obtained an archival FEROS spectrum of WASP-18 acquired on Sept. 9, 2010; in particular, we examinated the portion of spectrum around the $\mathrm{H}_{\alpha}$ and the Li doublet (6708 $\AA$ ). We estimated the rotational period of WASP-18 by deriving the projected rotational velocity $\mathrm{v} \sin i$. We measured the Gaussian full width at half maximum (FWHM) of the weak Fe lines around $\mathrm{Li}$ doublet equal to $F W H M=0.42 \AA$. We corrected this value by quadratically subtracting: a) $0.14 \AA$ of instrumental resolution; b) $1 \mathrm{~km} \mathrm{~s}^{-1}$ of micro turbulence; and c) $4 \mathrm{~km} \mathrm{~s}^{-1}$ of macro turbulence. The values of micro and macro turbulence are broadly consistent with those used by Doyle et al. (2013). We assumed that the axis of the planetary orbit aligned with the stellar rotational axis and an angle between orbital plane and line of sight $i=86 \pm 2.5 \mathrm{deg}$ to obtain a value of rotational velocity $v \sim 17.2 \pm 0.5 \mathrm{~km} \mathrm{~s}^{-1}$. Given a stellar radius of $1.29 R_{\odot}$, the rotational period is 3.7-3.9 days, which makes WASP-18 a moderate rotator. However, Doyle et al. (2013) report a slower $v \sin i$ $\left(10.9 \pm 0.7 \mathrm{~km} \mathrm{~s}^{-1}\right)$, which would give a period of $P \sim 6$ days. The discrepancy of our period (3.7-3.9 d) and the one inferred by Doyle et al. (2013) does not produce disagreement in the expected activity of WASP-18. For a star of the mass of WASP-18 and rotating in about four to six days, the expected X-ray luminosity should be $L_{\mathrm{X}} \geq 10^{29} \mathrm{erg} \mathrm{s}^{-1}$ (see Figs. 5 and 8 in Pizzolato et al. 2003).

\section{Results}

\subsection{No $X$-rays from WASP-18}

As stated in Sect. 2, WASP-18 is not detected in X-rays. The limiting count rate is $r_{\mathrm{lim}}=3.8 \times 10^{-5} \mathrm{ct} \mathrm{s}^{-1}$. By using PIMMS 
software, we can estimate a limiting flux and luminosity from the upper limit to the rate. We assumed a thermal spectrum with one temperature at $k T=0.5 \mathrm{keV}$, analogous to HD 189733 and the plasma temperatures typical of young Hyades, and a gas absorption equal to $N_{H}=1.5 \times 10^{20} \mathrm{~cm}^{-2}$. We obtained a limit unabsorbed flux of $3.8 \times 10^{-16} \mathrm{erg} \mathrm{s}^{-1} \mathrm{~cm}^{-2}$ in $0.3-8.0 \mathrm{keV}$ band, which corresponds to a luminosity of $L_{\mathrm{X}} \leq 4.5 \times 10^{26} \mathrm{erg} \mathrm{s}^{-1}$ at a distance of $100 \mathrm{pc}$. When using a softer spectrum $(k T=$ $0.3 \mathrm{keV})$, the limiting flux and luminosity would be $f_{\mathrm{X}}=5.9 \times$ $10^{-16} \mathrm{erg} \mathrm{s}^{-1} \mathrm{~cm}^{-2}$ and $L_{\mathrm{X}}=1.5 \times 10^{27} \mathrm{erg} \mathrm{s}^{-1}$, respectively. The $L_{\mathrm{X}}$ value are surprisingly low for a star whose age is estimated to be around 600 Myr like the Hyades. Late F stars in Hyades have a median luminosity of $L_{\mathrm{X}}=10^{29} \mathrm{erg} \mathrm{s}^{-1}$ with a dispersion of about 0.5 dex (Randich et al. 1998; Stern et al. 1995; Randich \& Schmitt 1995).

Even older $\mathrm{F}$ type stars can emit more X-ray luminosity than WASP-18. For comparison, we report the serendipitous detection in X-rays of the F6V type star HD 110450, in a 20ks exposure devoted to observe R Mus (P.I. N. Remage Evans, Pillitteri et al. in prep). HD 110450 is very similar to WASP-18: it is at $\sim 100$ pc from the Sun and has an age estimated between 2.1 Gyr and 3.9 Gyr (Casagrande et al. 2011; Holmberg et al. 2009). In X-rays, it has a PN rate of $20 \mathrm{ct} / \mathrm{ks}$ in the $0.3-8.0 \mathrm{keV}$ band and the best fit to the spectrum gives a temperature of $k T=0.2 \mathrm{keV}$ and $N_{\mathrm{H}}=3 \times 10^{21} \mathrm{~cm}^{-2}$, unabsorbed flux of $f_{\mathrm{X}}=2.5 \times 10^{-13} \mathrm{erg} \mathrm{s}^{-1} \mathrm{~cm}^{-2}$, and luminosity $L_{\mathrm{X}}=3.1 \times 10^{29} \mathrm{erg} \mathrm{s}^{-1}$. From this comparison, we conclude that if WASP-18 has the same age as of HD 110450, its low X-ray activity would still be at odds with that of a typical F-type star. The inactive F5 type star Procyon and the F7 type hot-Jupiter host $\tau$ Boo have X-ray luminosities of $\log L_{\mathrm{X}} \sim 28$ and $\log L_{\mathrm{X}}=28.8$ respectively. More interestingly, WASP-18 and $\tau$ Boo have similar rotational periods, 3.7-6 days for WASP-18, and 3.1-3.7 days for $\tau$ Boo (Catala et al. 2007). A similar level of X-ray activity should be observed because of the link between rotation and X-ray activity (Pallavicini et al. 1981; Pizzolato et al. 2003). However, the age of $\tau$ Boo is estimated to be around 2 Gyr, and its activity is perhaps boosted by magnetic SPI (Walker et al. 2008). WASP-18 stands out of the typical activity of similar mass stars.

Summarizing, WASP-18 is more than 2.5 orders of magnitude less luminous than Hyades, and about 2 orders of magnitude less active than the twin HD 110450, the inactive Procyon, and the similar planet host $\tau$ Boo. The absence of X-ray activity in WASP-18 agrees with the very low chromospheric activity reported by Knutson et al. (2010) and Miller et al. (2012), who report the non detection of this star in a $50 \mathrm{ks}$ stacked Swift exposure at $\log L_{\mathrm{Xlim}}=27.5$. Its low activity would suggest a much older age, of a few Gyr, because of the relationship between activity-rotation and age in main sequence stars, hence an age comparable to that of the Sun or older. Nevertheless, the isochronal age estimate of WASP-18 appears plausible given the agreement of several stellar models (Southworth et al. 2009). At odds with an age older than $2 \mathrm{Gyr}$ is also the moderately high rotational rate of WASP-18. The contradictory age estimates of WASP-18 imply that even an old age alone cannot explain the darkness of WASP-18 in X-rays. This points out to a role played by its close massive hot Jupiter.

\section{Discussion}

The first unexpected result we obtained with the $87 \mathrm{ks}$ Chandra exposure is the very low upper limit to the X-ray luminosity of

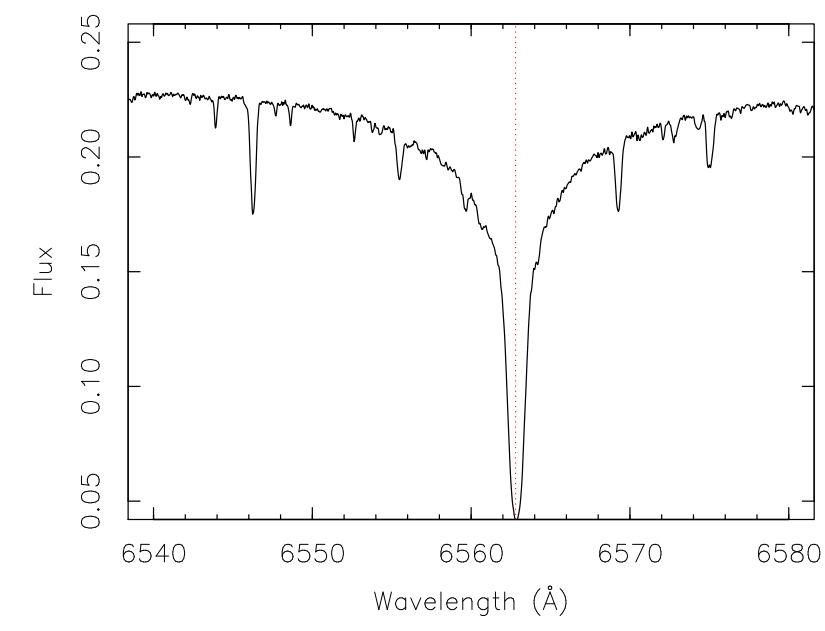

Fig. 2. Portion of FEROS spectrum of WASP-18 around $\mathrm{H} \alpha$ from the ESO archive of pipeline reduced spectra. This particular spectrum was taken on 19 Sept. 2010. We use arbitrary units for flux on the $Y$ axis. Absence of core emission in $\mathrm{H} \alpha$, and narrow lines support the idea that the star is not as young as Hyades and is a moderately high rotator.

WASP-18 $\left(L_{\text {Xlim }}=4.5 \times 10^{26} \mathrm{erg} / \mathrm{s}\right.$. The lack of X-ray and chromospheric activity of WASP-18 is inconsistent with its young age. Indeed the comparison with Hyades and with the similar stars, such as HD 110450, $\tau$ Boo, and Procyon, points to a much older age than given by Hellier et al. (2009). Optical spectra obtained at ESO Telescope with the FEROS spectrograph corfirm absence of activity and suggest an age similar to or older than that of the Sun (Soderblom 2010). Figure 2 shows the portion of one of the FEROS spectra around $\mathrm{H} \alpha$. The line is seen completely in absorption, with no signs of filling-in of the core that could be due to chromospheric activity. Moreover, the many weak absorption lines of $\mathrm{Fe}$ and other metals are quite narrow meaning a slow stellar rotation. Altogether, these features demonstrate the low activity of WASP-18 and would suggest an age closer to the Sun's. Using the empirical calibration given by Soderblom (2010), despite its limitations, and the value of $\log R_{\mathrm{HK}}^{\prime}=-5.43$ reported by Knutson et al. (2010) gives us an age $\tau \geq 2.7$ Gyr. The chromospheric activity indicator of WASP-18 is even below the average value of cluster M 67 (4.5 Gyr, Soderblom 2010) and the solar value.

However, the scenario becomes more puzzling when considering that WASP-18 shows Li absorption at $6708 \AA$, with a value typical of, or even stronger than values found in F-late stars of Hyades (Takeda et al. 2013) and M 67 (Pace et al. 2012). Figure 3 shows a portion of the spectrum of WASP-18 around the Li doublet lines at $6708 \AA$, and Fig. 4 shows the Li equivalent widths (EWs) vs. $T_{\text {eff }}$ given by Pace et al. (2012) and Takeda et al. (2013) for Hyades and the older M 67 cluster. For comparison we show the FEROS spectrum of $\tau$ Boo, which has no Li absorption. The Li absorption in WASP-18 suggests a younger age with respect to $\tau$ Boo or a different efficiency in the mixing mechanism among these two stars of similar effective temperatures and masses. In WASP-18 the equivalent width (EW) that we measured is $E W(L i)=46 \pm 2 \mathrm{~m} \AA$.

Compared to the values reported by Takeda et al. (2013) and Pace et al. (2012), WASP-18 has a slightly higher Li abundance. Lithium depletion is due to the convective mixing during the main sequence life of a star, so Li abundance is a rough indicator of youth in solar type stars with deep convective zone. In more massive stars, the mixing is less effective in bringing $\mathrm{Li}$ at the burning temperatures $\left(2.5 \times 10^{6} \mathrm{~K}\right)$. However, in mid 


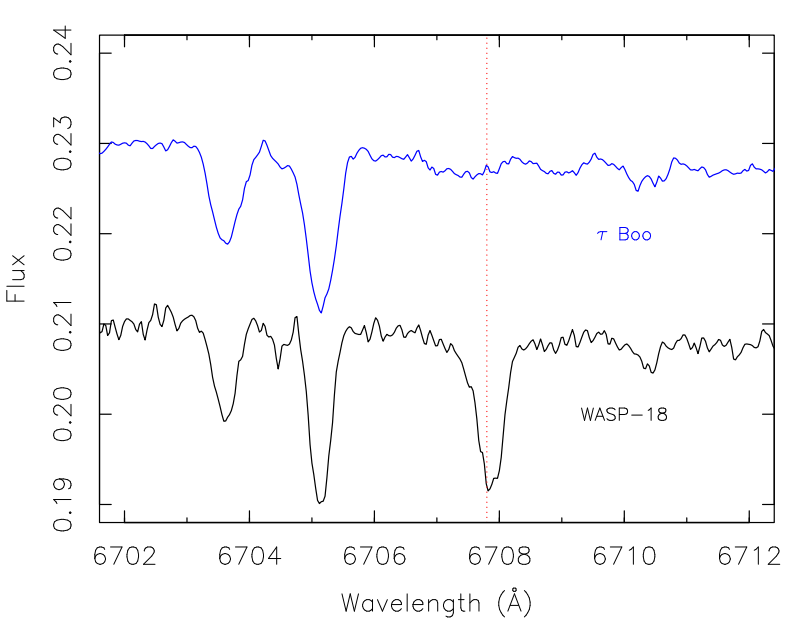

Fig. 3. Portion of the FEROS spectrum of WASP-18 around Li doublet at $6707.8 \AA$. This particular spectrum was taken on 19 Sept. 2010. We use arbitrary units for flux on $Y$ axis. For comparison we also plot the FEROS spectrum of $\tau$ Boo in the same spectral range. The spectrum of $\tau$ Boo has been scaled and shifted in wavelength by $+0.42 \AA$ (accounting for its radial velocity) for an easier comparison with WASP18. The Li feature is strong in WASP-18 and absent in $\tau$ Boo.

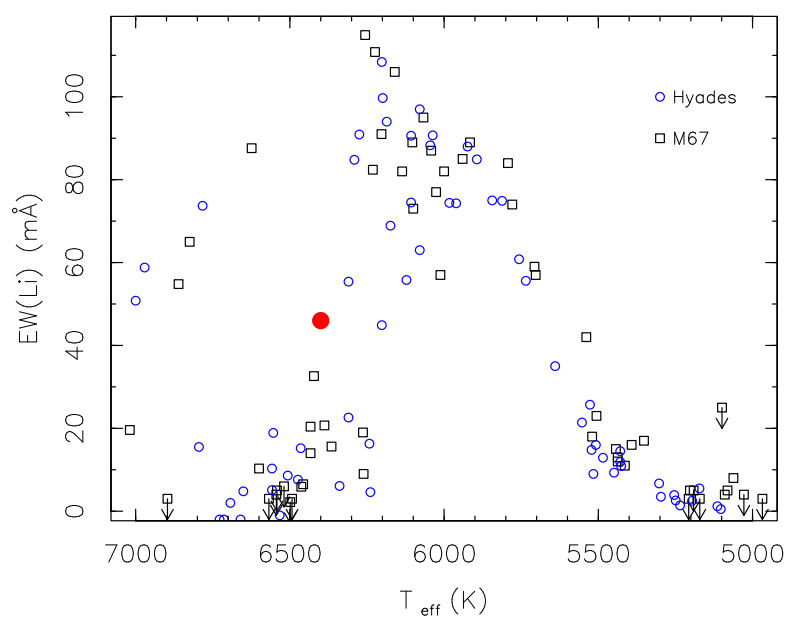

Fig. 4. EWs of Li vs. $T_{\text {eff }}$ for Hyades (open squares), M 67 (open circles) and WASP-18 (filled circle). The EW(Li) of WASP-18 is $E W=$ $46 \pm 2 \mathrm{~m} \AA$ and suggests a young age like Hyades, contradicting the absent activity of its corona and chromosphere.

F type stars Li abundance shows a significant dip that has still not been understood. The dip appears between $6700 \mathrm{~K}$ and $6200 \mathrm{~K}$ (see Fig. 3), with a steep edge on the hot side and a slower rise on the cool side. WASP-18 is in this range of temperatures $\left(T_{\text {eff }} \sim 6400 \mathrm{~K}\right)$ and should have a low Li abundance.

Israelian et al. (2004) claim that stars harboring hot Jupiters have lower $\mathrm{Li}$ abundance than single stars, and similar results were reported by Gonzalez (2008) and Delgado Mena et al. (2014). Bouvier (2008) qualitatively explains this result by tracing it back to the pre main Sequence (PMS) history of the angular momentum of the star+disk system. A long-lived circumstellar disk during PMS creates a slow stellar rotator with a strong shear at the base of the convective zone and a more efficient mixing that accelerates the Li burning. At the same time, a longlived disk offers more favorable conditions for the formation and the migration of exoplanets. WASP-18 has a significant Li abundance conflicting with the general pattern of $\mathrm{Li}$ in stars hosting hot Jupiters.

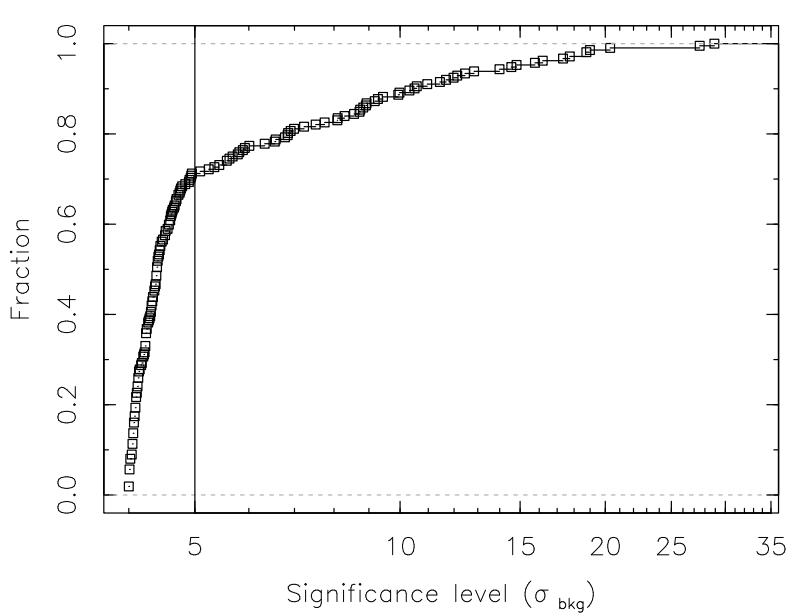

Fig. 5. Cumulative distribution of significance in $\sigma_{\mathrm{bkg}}$. Scale on $x$-axis is logarithmic. A break at a significance level $\sigma=5$ is noticed.

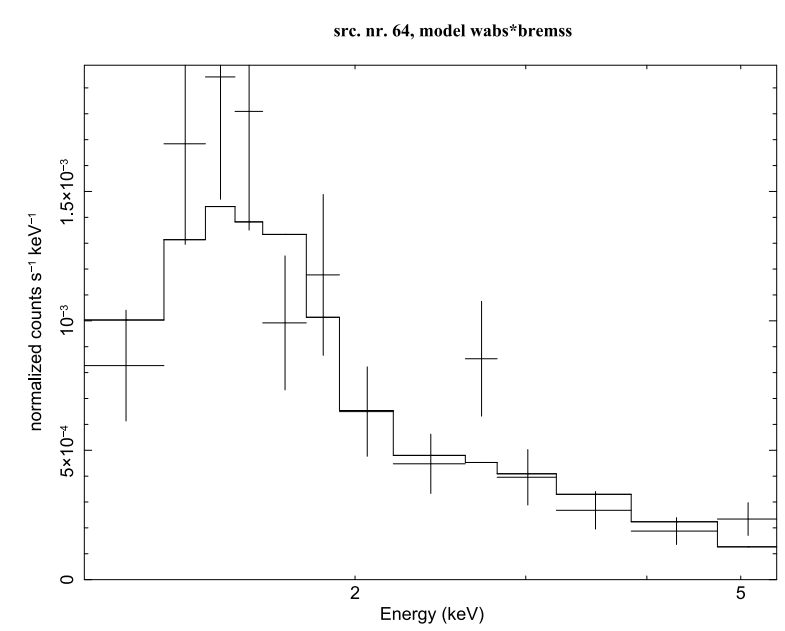

Fig. 6. X-ray spectrum and best fit model of src. 64 . A line at $\sim 2.5 \mathrm{keV}$ is noticed.

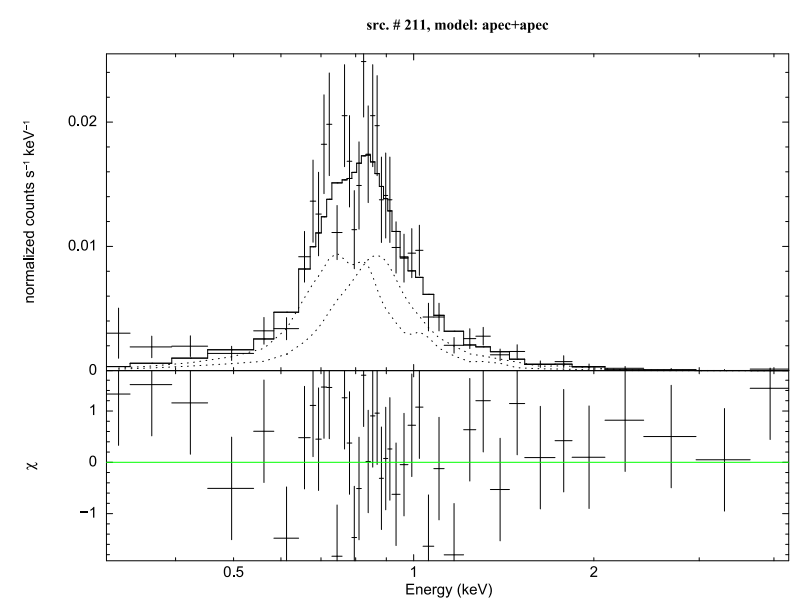

Fig. 7. X-ray spectrum and best fit model of the G8III/IV star HD 10210 (src. 211). A thermal model with two components $\left(k T_{1}=0.37 \mathrm{keV}\right.$, $\left.K T_{2}=0,75\right)$ is the best fit to the spectrum.

How can one reconcile the X-ray darkness, the absence of activity, an old age, and the "high" Li abundance in WASP-18? The solution to conflicting evidence could rely in the strong tidal interaction between the massive planet and its host star. We speculate that the tidal interaction in WASP-18 could interfere in 
Table 3. Properties of stars with hot Jupiter s with $T_{\text {eff }}$ in the range $6200-6600 \mathrm{~K}$.

\begin{tabular}{llllllllll}
\hline \hline Star & $\begin{array}{l}T_{\text {eff }} \\
\mathrm{K}\end{array}$ & $\begin{array}{l}R_{\text {star }} \\
R_{\odot}\end{array}$ & $\begin{array}{l}M_{\text {star }} \\
M_{\odot}\end{array}$ & $\begin{array}{l}M_{\text {planet }} \\
M_{\text {Jup }}\end{array}$ & $\begin{array}{l}\text { Separation } \\
\text { AU }\end{array}$ & $\log R_{\mathrm{HK}}^{\prime}$ & $\begin{array}{l}H_{P} \\
\mathrm{~km}\end{array}$ & $\begin{array}{l}H_{\mathrm{t}} \\
\mathrm{km}\end{array}$ & $H_{\mathrm{t}} / H_{P}$ \\
\hline WASP-18 & 6400 & 1.29 & 1.28 & 10.43 & 0.02047 & -5.43 & 419 & 498.3 & 1.189 \\
WASP-12 & 6300 & 1.599 & 1.35 & 1.404 & 0.02293 & -5.5 & 600.1 & 122.3 & 0.204 \\
WASP-14 & 6475 & 1.306 & 1.211 & 7.341 & 0.036 & -4.923 & 458.7 & 44 & 0.096 \\
XO-3 & 6429 & 1.377 & 1.213 & 11.79 & 0.0454 & -4.595 & 505.5 & 39.4 & 0.078 \\
HAT-P-7 & 6350 & 1.84 & 1.47 & 1.8 & 0.0379 & -5.018 & 735.5 & 37.2 & 0.051 \\
HAT-P-2 & 6290 & 1.64 & 1.36 & 8.74 & 0.0674 & -4.78 & 625.6 & 14.6 & 0.023 \\
Kepler-5 & 6297 & 1.793 & 1.374 & 2.114 & 0.05064 & -5.037 & 740.9 & 14.1 & 0.019 \\
HAT-P-14 & 6600 & 1.468 & 1.386 & 2.2 & 0.0594 & -4.855 & 516 & 3.4 & 0.007 \\
HAT-P-6 & 6570 & 1.46 & 1.29 & 1.057 & 0.05235 & -4.799 & 545.9 & 2.6 & 0.005 \\
Kepler-8 & 6213 & 1.486 & 1.213 & 0.603 & 0.0483 & -5.05 & 568.8 & 2.3 & 0.004 \\
WASP-17 & 6650 & 1.38 & 1.2 & 0.486 & 0.0515 & -5.331 & 530.7 & 1.1 & 0.002 \\
HAT-P-9 & 6350 & 1.32 & 1.28 & 0.67 & 0.053 & -5.092 & 434.7 & 1 & 0.002 \\
\hline WASP-19 & 5500 & 1.004 & 0.904 & 1.114 & 0.01616 & -4.66 & 308.5 & 55.2 & 0.179 \\
\hline
\end{tabular}

Notes. We list the effective temperatures, stellar masses and radii, planet-star separations, chromospheric activity indicator $\log R_{H K}^{\prime}$ (from Knutson et al. 2010), pressure scale heights $\left(H_{P}\right)$, tidal heights $\left(H_{t}\right)$, and ratio $H_{t} / H_{P}$ based on the formulae given by Cuntz et al. (2000). Stellar data are taken from the exoplanets.eu catalog and ordered by decreasing $H_{t} / H_{P}$ ratio. Data of WASP-19 are shown because of the planet-star separation very similar to WASP-18. WASP-18 is the only star with pressure scale height and tidal height of the same order of magnitude. The second highest $H_{t} / H_{P}$ ratio $(\sim 20 \%)$ is seen in WASP-12, which has low activity like WASP-18.

Table 4. Optical and IR counterparts of X-ray sources.

\begin{tabular}{|c|c|c|c|c|c|c|c|c|c|c|c|c|c|}
\hline \multirow{2}{*}{$\begin{array}{l}\# \\
20\end{array}$} & \multirow{2}{*}{$\begin{array}{c}\text { RA (deg) } \\
\text { X-ra } \\
24.29519\end{array}$} & Dec (deg) & No. & \multicolumn{3}{|c|}{ NED name } & \multirow{2}{*}{$\begin{array}{l}\text { RA(deg) } \\
24.29543\end{array}$} & \multicolumn{2}{|c|}{ DEC(deg) } & Type & $\begin{array}{l}\text { Magnitude } \\
\text { and filter }\end{array}$ & \multicolumn{2}{|c|}{$\begin{array}{r}\text { Separation } \\
\operatorname{arcsec}\end{array}$} \\
\hline & & -45.57693 & 62 & APMUKS(BJ & J) B013505.04-4549 & & & & & $\mathrm{G}$ & 18.99 & & 0.7 \\
\hline 22 & & -45.6 & 30 & MRSS 244-0 & 10213 & & 24.30153 & & & G & & & 0.7 \\
\hline 129 & 24.35544 & -45.6 & 6 & UKS(BJ & J) B013519.66-455 & & & & & G & & & 0.7 \\
\hline 131 & & -45 . & 5 & AP & 520 & & & & & $\mathrm{G}$ & & & 0.9 \\
\hline 184 & 24.4 & -45 . & 49 & $\mathrm{Ml}$ & 08106 & & & & 108 & $\mathrm{G}$ & & & 1.0 \\
\hline 186 & 24.50386 & -45.75107 & 70 & & J) В013555.40-460 & 17.6 & 24.5036 & -45.7 & 5096 & $\mathrm{G}$ & 19.55 & & 0.8 \\
\hline$\#$ & RA & Dec $\quad \mathrm{I}$ & RAJ2000 & DEJ2000 & 2MASS name & Jmag & e_Jmag & Hmag & e_Hmag & Kma & ig $\quad$ e_Kmag & Qflg & Sep. \\
\hline 20 & 4.29519 & -45.57693 & 24.295321 & & $01371087-4534374$ & 16.6 & & 15.9 & 0.13 & 15. & 0.16 & $\mathrm{BBC}$ & 0.6 \\
\hline 184 & 24.49494 & -45.65133 & 24.494989 & -45.65136 & 01375879-4539048 & 16.74 & 0.154 & 15.80 & 0.15 & 15. & 0.13 & BBB & 0.2 \\
\hline$\#$ & RA & DEC & Simb & $\operatorname{ad}$ ID OTYP & $\begin{array}{lll}\text { PE } & \text { pmRA } & \text { pmD }\end{array}$ & & Plx_VALUE & Z_V & ALUE & $\mathrm{B}$ & SP_T & ГYPE & Sep. \\
\hline 211 & 24.743367 & -45.77485 & HD 1 & 10210 & 48.35 & & 6.33 & & $8 \mathrm{E}-5$ & 9.02 & 8.08 & & 1.6 \\
\hline
\end{tabular}

Notes. The first part of the table lists the six NED counterparts, the second part lists the two 2MASS counterparts, the third part lists the only SIMBAD counterpart.

a significant way with the upper layers of the convective zone to the point of canceling out the magnetic activity and of reducing the mixing of the stellar material. Following Cuntz et al. (2000), we estimated a height of the tide of $H_{\mathrm{t}} \sim 498 \mathrm{~km}$. This value could still be a small fraction of the depth of the convective zone $\left(\sim 16 \% R_{*}\right.$, Houdek et al. 1999; Trampedach et al. 2013). However, the depths of the convective zone in stars of mass of WASP-18 or higher are very sensitive to the mass and temperature, and in this range of temperatures its calculation suffers of significant uncertainties (see Claret 2004). Among a sample of exoplanet host stars with similar effective temperatures, WASP-18 has the highest ratio between tide height and pressure scale height $\left(H_{t} / H_{P}\right)$. This ratio is on the order of 1.2 as shown in Table 3 , where we list the ratios $H_{\mathrm{t}} / H_{P}$ of a sample of stars with hot Jupiters with effective temperatures $T_{\text {eff }}$ in the range 6200-6600 K taken from Knutson et al. (2010). The $H_{\mathrm{t}} / H_{P}$ ratio takes into account the tidal effect due to the mass of the planet, its distance from the star (through $H_{\mathrm{t}}$ ), and the properties of the star $\left(T_{\text {eff }}\right.$, stellar mass and radius through $\left.H_{P}\right)$. We speculate that the tides on the stellar surface could influence the convective motions and the meridional circulation inside the convective layers to effectively reduce or nullify the mechanism of magnetic dynamo. The $H_{\mathrm{t}} / H_{P}$ ratio could be an empirical parameter of the efficiency of the planetary tide in reducing the shear within convective layers. The difference of $\mathrm{Li}$ in WASP-18 and $\tau$ Boo could be a manifestation of different tidal interactions in these two systems. It has been observed that in tidally locked binaries of Hyades, $\mathrm{Li}$ is more abundant than in single stars pointing to a role of tidal influence on the inner mixing of these stars (Thorburn et al. 1993; Deliyannis et al. 1994).

The existence of WASP-18b poses a strong constraint on the models of the dynamics of planets migrating inward. If the stellar age is in the range $2-4 \mathrm{Gyr}$, the inward planet migration has acted on a time scale of a few Gyr, not on a time scale of hundreds of Myr as derived by Brown et al. (2011). The orbital evolution of WASP-18 b has thus been slower than predicted by models of the orbital evolution of hot Jupiters. The low activity of the star also has consequences for the photo-evaporation of the planet and its lifetime. An X-ray and UV flux that is two orders of magnitude weaker than in other systems such as $\tau$ Boo and HD 189733, produces much less evaporation of the upper layers of the planetary atmosphere, making the process slower than in other active hosts of hot Jupiters (Penz \& Micela 2008; Sanz-Forcada et al. 2011). We expect that Roche-Lobe 
Table 5. Best fit models and parameters.

\begin{tabular}{|c|c|c|c|c|c|c|c|c|c|c|c|}
\hline \# & $\begin{array}{l}\text { Model } \\
\text { name }\end{array}$ & $\chi^{2}$ & d.o.f. & $N_{\mathrm{H}}$ & $\underset{\mathrm{cm}^{-2}}{\operatorname{err}\left(N_{\mathrm{H}}\right)}$ & $k \mathrm{~T} / \alpha$ & $\begin{array}{l}\operatorname{err}(K T / \alpha) \\
\mathrm{keV} /-\end{array}$ & Norm & $\underset{m^{-5}}{\operatorname{err}(\text { Norm) }}$ & & $\underset{\operatorname{erg~s}^{-1} \mathrm{~cm}^{-2}}{\operatorname{Flux}}$ \\
\hline 8 & Abs+Pow & 2.82 & 6 & 0.10 & 0.22 & 1.8 & 0.5 & $4.2 \mathrm{e}-06$ & $2.0 \mathrm{e}-06$ & & $2.0 \mathrm{e}-14$ \\
\hline 20 & $\mathrm{Abs}+\mathrm{Pow}$ & 4.13 & 4 & 0.35 & 0.50 & 0.51 & 0.5 & $1.8 \mathrm{e}-06$ & $1.3 \mathrm{e}-06$ & & $4.0 \mathrm{e}-14$ \\
\hline 22 & Abs+Pow & 4.13 & 4 & 0.35 & 0.5 & 0.5 & 0.5 & $1.8 \mathrm{e}-6$ & $1.2 \mathrm{e}-6$ & & $5.4 \mathrm{e}-14$ \\
\hline 57 & Abs+Pow & 9.7 & 5 & 0.4 & 0.3 & 3.5 & 0.8 & $1.7 \mathrm{e}-05$ & $1.0 \mathrm{e}-05$ & & $1.7 \mathrm{e}-14$ \\
\hline 64 & Abs+Brems & 13.0 & 10 & 0.61 & 0.27 & 4.9 & 2.9 & $1.33 \mathrm{e}-05$ & $4.1 \mathrm{e}-06$ & & $3.5 \mathrm{e}-14$ \\
\hline 90 & Abs+Pow & 6.91 & 3 & 4.6 & 1.9 & 2.9 & 1.0 & $4.3 e-05$ & $5.8 \mathrm{e}-05$ & & $1.8 \mathrm{e}-14$ \\
\hline 104 & Abs+Pow & 4.51 & 6 & 0.13 & 0.16 & 1.9 & 0.4 & $5.5 \mathrm{e}-06$ & $2.1 \mathrm{e}-06$ & & $2.2 \mathrm{e}-14$ \\
\hline 135 & $\mathrm{Abs}+\mathrm{Pow}$ & 0.45 & 1 & 1.2 & 0.8 & 2.1 & 0.8 & $4.4 \mathrm{e}-06$ & $4.8 \mathrm{e}-06$ & & $9.6 e-15$ \\
\hline 145 & Abs+Pow & 0.96 & 1 & 0.02 & 0.20 & 1.8 & 0.5 & $1.6 \mathrm{e}-06$ & $0.9 \mathrm{e}-06$ & & $9.0 \mathrm{e}-15$ \\
\hline 182 & Abs+Pow & 2.35 & 2 & 0.0 & 0.2 & 1.6 & 0.5 & $1.7 \mathrm{e}-06$ & $0.9 \mathrm{e}-06$ & & $1.2 \mathrm{e}-14$ \\
\hline 190 & Abs+Pow & 0.22 & 3 & 0.0 & 0.23 & 1.5 & 0.5 & $4.3 \mathrm{e}-06$ & $2.3 \mathrm{e}-06$ & & $3.1 \mathrm{e}-14$ \\
\hline$\#$ & Model & $\chi^{2}$ & d.o.f. & $k T_{1}$ & $\operatorname{err}\left(K T_{!}\right)$ & $k T_{2}$ & $\operatorname{err}\left(K T_{2}\right)$ & Norm1 & err(Norm1) & err(Norm2) & flux \\
\hline 211 & Apec+Apec & 41.1 & 36 & 0.37 & 0.06 & 0.75 & 0.1 & $4.1 \mathrm{e}-5$ & $2.0 \mathrm{e}-5$ & $1.7 e-5$ & $9.1 \mathrm{e}-14$ \\
\hline
\end{tabular}

Notes. We used an absorbed power law as model (Abs+Pow) for all but two cases, where we used absorbed bremsstrahlung (src. \# 64, Abs+Brems) and two thermal models (src. \# 211, Apec+Apec). Errors are given at the $1 \sigma$ level.

enhancement of the evaporation (Erkaev et al. 2007) should not be important in WASP-18b, given its mass $\left(\sim 10.4 M_{\text {jup }}\right)$.

The absence of a significant corona explains why the star is dark in X-rays. WASP-18 demonstrates that SPI of tidal and magnetic origin must depend on both stellar structure and evolutionary stage. A different example of SPI in a star with a very close-in hot Jupiter is given by WASP-19. Like WASP-18, WASP-19 has a close-in hot Jupiter that orbits in less than one day. However, WASP-19 is a K type star with a deep convective zone and has a planet of almost the mass of Jupiter. WASP-19 shows high chromospheric emission, at a level similar to HD 189733 (Knutson et al. 2010). The $H_{\mathrm{t}} / H_{P}$ ratio in WASP-19 is $18 \%$ and the height of the tide is $55.2 \mathrm{~km}$, a very small fraction of the depth of the convective zone in this star. We argue that the different stellar structures of WASP-19 and WASP-18 probably result in different dynamo strengths and coronal emission. We argue that in the case of WASP-19, the tidal interaction cannot significantly affect the motions of material inside the convective zone as in the case of WASP-18. As a consequence, a magnetic dynamo can still be established, and magnetic SPI is at work in this system enhancing the overall activity of WASP-19. With opposite effects, the tidal interaction of WASP-18b takes over the magnetic influence and suppresses the magnetic dynamo of WASP-18.

In summary, the factors that lead to SPI not only are a function of planet-star separation and planet/star mass ratio, but also rely on the inner structure of the parent star, the efficiency of its dynamo, its age, and the strength of a planetary magnetic field.

\section{Summary and conclusions}

With the aim of detecting the effects of star-planet interaction at high energies, we analysed a $87 \mathrm{ks}$ deep Chandra observation pointed toward the star with hot Jupiter WASP-18. We do not find any X-ray emission from the star at a level above $L_{\mathrm{X}}=4.5 \times 10^{26} \mathrm{erg} \mathrm{s}^{-1}$. The star is at least 2.5 orders of magnitude less luminous in $\mathrm{X}$-rays than analog $\mathrm{F}$ late stars in Hyades, and main sequence stars like $\tau$ Boo, the 4 Gyr old star HD 110450 and Procyon, which is at the very end of the main sequence or already post main sequence. The absence of X-ray activity agrees with the low chromospheric activity reported by Knutson et al. (2010), with the absence of reversal core emission in the $\mathrm{H} \alpha$ and $\mathrm{Ca} \mathrm{H}$ and $\mathrm{K}$ lines. These facts strongly conflict with the estimate of the age from fitting to isochrones (600 Myr) given by Hellier et al. (2009) and Southworth et al. (2009), and with the strong Li absorption observed in optical spectra and would suggest an activity level that is more consistent with a solar age.

A stellar age of a few Gyr puts strong constraints on the evolution model of the planet. In particular, it implies that the inward planet migration took place on a time scale of a few Gyr, not on a time scale of hundreds of Myr as assessed by Brown et al. (2011). The orbital evolution of WASP-18 b may therefore have been slower than predicted by models.

The absence of X-ray activity in the star also indicates a null efficiency of the magnetic dynamo. In these conditions, magnetic SPI is not at work, but rather, a strong tidal influence from the massive hot Jupiter can play a major role in determining the outer stellar structure and activity of WASP-18. To reconcile the strong $\mathrm{Li}$ absorption with an absent activity, we suggest a scenario in which the tidal interaction of the massive planet has modified the inner stellar mixing, thus preventing or at least reducing the Li burning. At the same time, the upper layers of the thin convective zone expected at this stellar mass are profoundly altered by tidal stresses. In a sample of stars with hot Jupiters in the same range of effective temperature, WASP-18 is the only object to show a tide height, induced by its planet, that is higher than the gas pressure scale height. The motions induced by tidal SPI could reduce the shear within the convective zone, hampering the creation of a magnetic dynamo and thus the outer corona and the production of X-rays. The same tides could be responsible for reducting the mixing efficiency in the inner stellar layers resulting in a higher Li abundance than observed in stars of similar mass, like $\tau$ Boo. This hypothesis requires detailed simulations in the framework of two-body interaction and modification of the stellar structure in binary systems. Our results can be a stimulus to understanding any of these effects, and then highlight the uniqueness of WASP-18 among systems with hot Jupiters.

We find 212 X-ray sources in the ACIS image. We briefly discuss their characteristics in the Appendix.

Acknowledgements. I.P. and S.J.W. are grateful to Dr. S. Saar for his comments on this paper. I.P. acknowledges financial support from the European Union under the project "Astronomy Fellowships in Italy" (AstroFit). S.J.W. was supported by NASA contract NAS8-03060. V.A. is grateful to Dr. R. Trampedach for the discussion about the models of stellar convection. V.A. acknowledges funding for the Stellar Astrophysics Centre provided by The Danish National Research Foundation; the research is supported by the ASTERISK project (ASTERoseismic Investigations with SONG and Kepler) funded by the European Research Council (Grant agreement no. 267864). 


\section{Appendix A: Nature of the X-ray sources}

In the ACIS image we have detected 212 X-ray sources with significance $>4 \sigma$. We cross matched their spatial positions with Simbad, NED, and 2MASS catalogs within a positional separation of $2^{\prime \prime}$, obtaining seven matches that are listed in Table 4. All NED matches are galaxies, and the X-ray emission could be associated to the AGNs at their center. One match is the giant/sub-giant G8III/IV star (HD 10210, src \# 211) with $V=$ 8.08. The two matches in 2MASS are again two galaxies in the NED catalog.

We are left with 205 X-ray sources without matches in the above catalogs. Most of these sources are faint as shown by the cumulative distribution of the significance values (Fig. 5). The cumulative distribution has a change of slope at about $\sigma=5$, marking the brighter sample from the rest of the sources. The number of unidentified sources with $\sigma>5$ is 55 , or the $27 \%$ of the total sample. A number of them could be distant AGNs.

For the brightest sources we did a model best fit to the spectra with one absorbed powerlaw, suited in the case of AGNs and well describing the featureless spectra that we observe in these sources. In the bright source \# 64 we find a good best fit with an absorbed bremsstrahlung, but the presence of a line at $\sim 2.5 \mathrm{keV}$ is also noticed.

The G8III/IV type star HD 10210 is also detected as the brightest in the sample (source \# 211), The best fit of the spectrum of HD 10210 has two temperatures at $k T=0.37$ and $k T=0.75$, with the cool component weighting twice the hot component in the spectrum. Overall, the spectrum is similar to that of a mid-active main sequence star, and its X-ray luminosity is $L_{\mathrm{X}} \sim 1.1 \times 10^{29} \mathrm{erg} / \mathrm{s}$. Detecting X-ray emission in a evolved star off of the main sequence is worth to be noticed,

\section{References}

Baliunas, S. L., Donahue, R. A., Soon, W. H., et al. 1995, ApJ, 438, 269 Bouvier, J. 2008, A\&A, 489, L53

Brown, D. J. A., Cameron, A. C., Hall, C., Hebb, L., \& Smalley, B. 2011, MNRAS, 415, 605

Casagrande, L., Schönrich, R., Asplund, M., et al. 2011, A\&A, 530, A138

Catala, C., Donati, J.-F., Shkolnik, E., Bohlender, D., \& Alecian, E. 2007, MNRAS, 374, L42

Claret, A. 2004, A\&A, 424, 919

Claret, A. 2005, A\&A, 440, 647

Claret, A. 2006, A\&A, 453, 769

Claret, A. 2007, A\&A, 467, 1389

Cuntz, M., Saar, S. H., \& Musielak, Z. E. 2000, ApJ, 533, L151

Damiani, F., Maggio, A., Micela, G., \& Sciortino, S. 1997a, ApJ, 483, 350

Damiani, F., Maggio, A., Micela, G., \& Sciortino, S. 1997b, ApJ, 483, 370

Damiani, F., Flaccomio, E., Micela, G., et al. 2003, ApJ, 588, 1009

Delgado Mena, E., Israelian, G., González Hernández, J. I., et al. 2014, A\&A, 562, A92
Deliyannis, C. P., King, J. R., Boesgaard, A. M., \& Ryan, S. G. 1994, ApJ, 434, L71

Demarque, P., Woo, J.-H., Kim, Y.-C., \& Yi, S. K. 2004, ApJS, 155, 667

Doyle, A. P., Smalley, B., Maxted, P. F. L., et al. 2013, MNRAS, 428, 3164

Eldridge, J. J., \& Tout, C. A. 2004, MNRAS, 353, 87

Erkaev, N. V., Kulikov, Y. N., Lammer, H., et al. 2007, A\&A, 472, 329

Fares, R., Donati, J.-F., Moutou, C., et al. 2010, MNRAS, 406, 409

Giardino, G., Favata, F., Pillitteri, I., et al. 2007, A\&A, 475, 891

Gonzalez, G. 2008, MNRAS, 386, 928

Hellier, C., Anderson, D. R., Collier Cameron, A., et al. 2009, Nature, 460, 1098 Holmberg, J., Nordström, B., \& Andersen, J. 2009, A\&A, 501, 941

Houdek, G., Balmforth, N. J., Christensen-Dalsgaard, J., \& Gough, D. O. 1999 A\&A, 351, 582

Ip, W.-H., Kopp, A., \& Hu, J.-H. 2004, ApJ, 602, L53

Israelian, G., Santos, N. C., Mayor, M., \& Rebolo, R. 2004, A\&A, 414, 601

Kashyap, V. L., Drake, J. J., \& Saar, S. H. 2008, ApJ, 687, 1339

Knutson, H. A., Howard, A. W., \& Isaacson, H. 2010, ApJ, 720, 1569

Krejčová, T., \& Budaj, J. 2012, A\&A, 540, A82

Melo, C., Santos, N. C., Pont, F., et al. 2006, A\&A, 460, 251

Miller, B. P., Gallo, E., Wright, J. T., \& Dupree, A. K. 2012, ApJ, 754, 137

Pace, G., Castro, M., Meléndez, J., Théado, S., \& do Nascimento, Jr., J.-D. 2012, A\&A, 541, A150

Pallavicini, R., Golub, L., Rosner, R., et al. 1981, ApJ, 248, 279

Penz, T., \& Micela, G. 2008, A\&A, 479, 579

Pillitteri, I., Wolk, S. J., Cohen, O., et al. 2010, ApJ, 722, 1216

Pillitteri, I., Günther, H. M., Wolk, S. J., Kashyap, V. L., \& Cohen, O. 2011, ApJ, 741, L18

Pillitteri, I., Wolk, S. J., Lopez-Santiago, J., et al. 2014, ApJ, 785, 145

Pizzolato, N., Maggio, A., Micela, G., Sciortino, S., \& Ventura, P. 2003, A\&A, 397, 147

Pols, O. R., Schröder, K.-P., Hurley, J. R., Tout, C. A., \& Eggleton, P. P. 1998, MNRAS, 298, 525

Poppenhaeger, K., \& Schmitt, J. H. M. M. 2011, ApJ, 735, 59

Poppenhaeger, K., \& Wolk, S. J. 2014, A\&A, 565, L1

Poppenhaeger, K., Robrade, J., \& Schmitt, J. H. M. M. 2010, A\&A, 515, A98

Poppenhaeger, K., Schmitt, J. H. M. M., \& Wolk, S. J. 2013, ApJ, 773, 62

Randich, S., \& Schmitt, J. H. M. M. 1995, A\&A, 298, 115

Randich, S., Singh, K. P., Simon, T., Drake, S. A., \& Schmitt, J. H. M. M. 1998, A\&A, 337, 372

Saar, S. H., Cuntz, M., \& Shkolnik, E. 2004, in Stars as Suns : Activity, Evolution and Planets, eds. A. K. Dupree, \& A. O. Benz, IAU Symp., 219, 355

Sanz-Forcada, J., Micela, G., Ribas, I., et al. 2011, A\&A, 532, A6

Schröter, S., Czesla, S., Wolter, U., et al. 2011, A\&A, 532, A3

Shkolnik, E., Walker, G. A. H., \& Bohlender, D. A. 2003, ApJ, 597, 1092

Skumanich, A. 1972, ApJ, 171, 565

Soderblom, D. R. 2010, ARA\&A, 48, 581

Southworth, J., Hinse, T. C., Dominik, M., et al. 2009, ApJ, 707, 167

Stern, R. A., Schmitt, J. H. M. M., \& Kahabka, P. T. 1995, ApJ, 448, 683

Takeda, Y., Honda, S., Ohnishi, T., et al. 2013, PASJ, 65, 53

Thorburn, J. A., Hobbs, L. M., Deliyannis, C. P., \& Pinsonneault, M. H. 1993, ApJ, 415, 150

Torres, G., Winn, J. N., \& Holman, M. J. 2008, ApJ, 677, 1324

Trampedach, R., Asplund, M., Collet, R., Nordlund, A., \& Stein, R. F. 2013, ApJ, 769, 18

Vaughan, A. H., \& Preston, G. W. 1980, PASP, 92, 385

Walker, G. A. H., Croll, B., Matthews, J. M., et al. 2008, A\&A, 482, 691

Wilson, O. C. 1966, ApJ, 144, 695

Yi, S., Demarque, P., Kim, Y.-C., et al. 2001, ApJS, 136, 417 
Table 2. continued.

\begin{tabular}{|c|c|c|c|c|c|c|c|c|c|c|}
\hline Num. & $\begin{array}{l}\text { RA (J2000) } \\
\text { deg }\end{array}$ & $\begin{array}{l}\text { Dec (J2000) } \\
\text { deg }\end{array}$ & $\begin{array}{l}\text { Pos. err } \\
\text { arcsec }\end{array}$ & $\begin{array}{l}\text { Off-axis } \\
\text { arcmin }\end{array}$ & $\begin{array}{r}\text { Significance } \\
\sigma_{\mathrm{bkg}}\end{array}$ & \multicolumn{2}{|c|}{ cts } & Rate & $\begin{array}{l}\text { Rate err. } \\
\mathrm{ks}^{-1}\end{array}$ & $\begin{array}{l}\text { Exp. time } \\
\text { ks }\end{array}$ \\
\hline 11 & 24.27388 & -45.60273 & 1.3 & 5.93 & 5.34 & 13.81 & 5.74 & 0.183 & 0.076 & 75.59 \\
\hline 12 & 24.27401 & -45.62748 & 2 & 4.75 & 4.08 & 12.11 & 5.41 & 0.151 & 0.067 & 80.22 \\
\hline 13 & 24.27558 & -45.60022 & 1.5 & 6.02 & 4.12 & 9.84 & 6.11 & 0.131 & 0.081 & 75.09 \\
\hline 14 & 24.27661 & -45.64517 & 1.4 & 3.95 & 4.41 & 9.89 & 4.44 & 0.121 & 0.054 & 82.04 \\
\hline 15 & 24.28193 & -45.4945 & 9 & 11.83 & 4.02 & 57.15 & 19.28 & 1.872 & 0.632 & 30.52 \\
\hline 16 & 24.28404 & -45.64753 & 0.5 & 3.62 & 4.12 & 4.48 & 3.39 & 0.055 & 0.042 & 80.82 \\
\hline 17 & 24.28427 & -45.60261 & 1.4 & 5.71 & 4.09 & 9.53 & 4.28 & 0.13 & 0.058 & 73.18 \\
\hline 18 & 24.28557 & -45.65013 & 0.5 & 3.47 & 11.98 & 33.92 & 9.76 & 0.455 & 0.131 & 74.6 \\
\hline 19 & 24.29219 & -45.59813 & 1.1 & 5.8 & 4.25 & 8.3 & 4.95 & 0.115 & 0.069 & 72.25 \\
\hline 20 & 24.29519 & -45.57693 & 1.2 & 6.93 & 14.82 & 69.33 & 9.94 & 1.005 & 0.144 & 68.98 \\
\hline 21 & 24.30081 & -45.63905 & 0.4 & 3.5 & 4.37 & 5.03 & 2.97 & 0.062 & 0.036 & 81.43 \\
\hline 22 & 24.3018 & -45.60976 & 0.7 & 5 & 27.51 & 136.87 & 12.3 & 1.893 & 0.17 & 72.3 \\
\hline 23 & 24.30209 & -45.65241 & 0.5 & 2.86 & 4.4 & 5.11 & 4.07 & 0.061 & 0.049 & 83.72 \\
\hline 24 & 24.30535 & -45.60159 & 1.1 & 5.39 & 4.4 & 8.7 & 5.11 & 0.115 & 0.068 & 75.5 \\
\hline 25 & 24.30621 & -45.64441 & 0.6 & 3.1 & 4.73 & 6.6 & 3.93 & 0.082 & 0.049 & 80.17 \\
\hline 26 & 24.30714 & -45.64659 & 0.4 & 2.98 & 5.92 & 8.49 & 4.41 & 0.109 & 0.056 & 78.28 \\
\hline 27 & 24.30823 & -45.61779 & 0.8 & 4.45 & 4.2 & 6.52 & 4.28 & 0.08 & 0.053 & 81.22 \\
\hline 28 & 24.31052 & -45.51348 & 3.8 & 10.47 & 6.85 & 55.06 & 16.95 & 1.234 & 0.38 & 44.61 \\
\hline 29 & 24.31374 & -45.59013 & 1.4 & 5.94 & 8.09 & 30.25 & 9.07 & 0.414 & 0.124 & 73.03 \\
\hline 30 & 24.31829 & -45.66284 & 0.5 & 1.93 & 4.09 & 4.54 & 3.56 & 0.054 & 0.042 & 84.49 \\
\hline 31 & 24.31834 & -45.6034 & 1.1 & 5.12 & 4.02 & 7.59 & 4.3 & 0.095 & 0.054 & 79.69 \\
\hline 32 & 24.3201 & -45.5065 & 3.8 & 10.82 & 6.33 & 48.21 & 13.57 & 1.293 & 0.364 & 37.29 \\
\hline 33 & 24.32353 & -45.65841 & 0.6 & 2 & 8.72 & 20.88 & 7.07 & 0.247 & 0.084 & 84.44 \\
\hline 34 & 24.32374 & -45.64749 & 0.6 & 2.56 & 4 & 5.16 & 2.97 & 0.061 & 0.035 & 84.28 \\
\hline 35 & 24.32418 & -45.55242 & 2 & 8.07 & 8.91 & 40.28 & 11.74 & 0.613 & 0.179 & 65.74 \\
\hline 36 & 24.32638 & -45.64832 & 0.5 & 2.46 & 4.32 & 5.07 & 4.12 & 0.06 & 0.049 & 84.37 \\
\hline 37 & 24.328 & -45.57583 & 1.4 & 6.66 & 8.74 & 33.24 & 9.82 & 0.469 & 0.139 & 70.89 \\
\hline 38 & 24.32838 & -45.55383 & 2 & 7.96 & 9.18 & 42.02 & 12.16 & 0.67 & 0.194 & 62.74 \\
\hline 39 & 24.32917 & -45.63721 & 0.5 & 3.04 & 4.33 & 5.12 & 3.94 & 0.063 & 0.048 & 81.61 \\
\hline 40 & 24.33661 & -45.62566 & 0.5 & 3.65 & 4.25 & 4.94 & 3.58 & 0.061 & 0.044 & 80.88 \\
\hline 41 & 24.33933 & -45.65263 & 0.5 & 2.04 & 4.24 & 4.83 & 3.64 & 0.058 & 0.044 & 82.64 \\
\hline 42 & 24.33979 & -45.60188 & 1.4 & 5.05 & 4.06 & 9.43 & 4.37 & 0.118 & 0.055 & 79.83 \\
\hline 43 & 24.34135 & -45.64186 & 0.5 & 2.66 & 4.24 & 4.81 & 3.71 & 0.059 & 0.046 & 80.91 \\
\hline 44 & 24.34422 & -45.59913 & 1.1 & 5.2 & 4.69 & 9.13 & 4.89 & 0.125 & 0.067 & 73.02 \\
\hline 45 & 24.34877 & -45.64658 & 0.4 & 2.34 & 5.81 & 8.1 & 4.22 & 0.107 & 0.056 & 75.47 \\
\hline 46 & 24.34891 & -45.63556 & 0.5 & 3 & 4.11 & 4.48 & 3.49 & 0.055 & 0.043 & 81.55 \\
\hline 47 & 24.35358 & -45.53544 & 3.6 & 9.01 & 4.06 & 20.91 & 8.35 & 0.393 & 0.157 & 53.19 \\
\hline 48 & 24.3545 & -45.61487 & 0.9 & 4.25 & 5.88 & 12.79 & 5.04 & 0.157 & 0.062 & 81.47 \\
\hline 49 & 24.35542 & -45.64032 & 0.5 & 2.72 & 4.1 & 4.38 & 3.27 & 0.055 & 0.041 & 79.98 \\
\hline 50 & 24.35714 & -45.64254 & 0.4 & 2.6 & 4.95 & 5.99 & 4.03 & 0.076 & 0.051 & 78.56 \\
\hline 51 & 24.3657 & -45.62035 & 0.8 & 3.96 & 4.08 & 6 & 3.29 & 0.075 & 0.041 & 79.69 \\
\hline 52 & 24.37347 & -45.6293 & 0.5 & 3.51 & 4.56 & 5.17 & 4.29 & 0.063 & 0.052 & 81.74 \\
\hline 53 & 24.37466 & -45.59796 & 12.1 & 5.35 & 4.18 & 78.92 & 25.07 & 1.011 & 0.321 & 78.04 \\
\hline 54 & 24.38382 & -45.616 & 0.8 & 4.4 & 4.66 & 7.04 & 5 & 0.09 & 0.064 & 78.44 \\
\hline 55 & 24.38498 & -45.58196 & 1.4 & 6.38 & 4.6 & 10.96 & 5.16 & 0.15 & 0.071 & 72.95 \\
\hline 56 & 24.38749 & -45.58205 & 1.4 & 6.4 & 4.01 & 9.09 & 4.33 & 0.125 & 0.06 & 72.59 \\
\hline 57 & 24.39812 & -45.56449 & 1.1 & 7.53 & 18.76 & 91.19 & 10.84 & 1.53 & 0.182 & 59.59 \\
\hline 58 & 24.42006 & -45.58314 & 1.5 & 6.8 & 4.13 & 8.38 & 5.07 & 0.178 & 0.108 & 47.15 \\
\hline 59 & 24.29277 & -45.79012 & 3.2 & 6.73 & 5.68 & 27.83 & 9.46 & 0.441 & 0.15 & 63.12 \\
\hline 60 & 24.2985 & -45.77849 & 6.6 & 5.99 & 4.05 & 35.21 & 12.36 & 0.512 & 0.18 & 68.72 \\
\hline 61 & 24.2994 & -45.73798 & 1 & 3.81 & 9.94 & 31.77 & 9.36 & 0.394 & 0.116 & 80.62 \\
\hline 62 & 24.30758 & -45.74881 & 2.5 & 4.2 & 4.24 & 15.75 & 6.3 & 0.211 & 0.084 & 74.72 \\
\hline 63 & 24.32088 & -45.70922 & 0.5 & 1.9 & 4.1 & 4.35 & 3.45 & 0.052 & 0.041 & 84.03 \\
\hline 64 & 24.32203 & -45.7925 & 1.2 & 6.53 & 28.91 & 175 & 14.52 & 2.534 & 0.21 & 69.05 \\
\hline 65 & 24.32337 & -45.76748 & 1.1 & 5.05 & 4.15 & 7.41 & 4.21 & 0.097 & 0.055 & 76.07 \\
\hline 66 & 24.32379 & -45.72558 & 0.6 & 2.65 & 15.77 & 43.82 & 6.97 & 0.577 & 0.092 & 75.91 \\
\hline 67 & 24.33132 & -45.78434 & 1.3 & 5.98 & 10.58 & 33.65 & 6.58 & 0.467 & 0.091 & 72.03 \\
\hline 68 & 24.33234 & -45.74109 & 0.5 & 3.42 & 4.13 & 4.42 & 3.2 & 0.056 & 0.041 & 78.25 \\
\hline 69 & 24.33327 & -45.72204 & 0.5 & 2.31 & 4.53 & 5.19 & 4.29 & 0.064 & 0.053 & 80.89 \\
\hline 70 & 24.33356 & -45.72145 & 2.6 & 2.27 & 4.07 & 15.28 & 6.14 & 0.194 & 0.078 & 78.6 \\
\hline 71 & 24.33479 & -45.68913 & 0.5 & 0.71 & 4.3 & 5.04 & 3.75 & 0.061 & 0.045 & 83.25 \\
\hline 72 & 24.33661 & -45.70409 & 0.6 & 1.26 & 7.23 & 14.93 & 5.53 & 0.176 & 0.065 & 84.63 \\
\hline 73 & 24.33712 & -45.69782 & 0.8 & 0.93 & 4.1 & 6.32 & 3.32 & 0.074 & 0.039 & 84.92 \\
\hline
\end{tabular}


Table 2. continued.

\begin{tabular}{|c|c|c|c|c|c|c|c|c|c|c|}
\hline Num. & $\begin{array}{l}\text { RA (J2000) } \\
\text { deg }\end{array}$ & $\begin{array}{l}\text { Dec (J2000) } \\
\text { deg }\end{array}$ & $\begin{array}{l}\text { Pos. err } \\
\text { arcsec }\end{array}$ & $\begin{array}{l}\text { Off-axis } \\
\text { arcmin }\end{array}$ & $\begin{array}{r}\text { Significance } \\
\sigma_{\mathrm{bkg}} \\
\end{array}$ & $\begin{array}{r}\text { Counts } \\
\mathrm{c}\end{array}$ & Cts err. & Rate & $\begin{array}{l}\text { Rate err. } \\
\mathrm{ks}^{-1}\end{array}$ & $\begin{array}{l}\text { Exp. time } \\
\text { ks }\end{array}$ \\
\hline 74 & 24.33959 & -45.70337 & 0.5 & 1.17 & 4.01 & 4.37 & 3.37 & 0.052 & 0.04 & 84.66 \\
\hline 75 & 24.34094 & -45.72871 & 0.5 & 2.62 & 4.42 & 5.07 & 4.08 & 0.063 & 0.051 & 80.17 \\
\hline 76 & 24.3419 & -45.69908 & 0.6 & 0.89 & 5.57 & 8.69 & 4.59 & 0.102 & 0.054 & 84.89 \\
\hline 77 & 24.3439 & -45.80344 & 1.5 & 7.08 & 4.05 & 8.24 & 4.25 & 0.134 & 0.069 & 61.39 \\
\hline 78 & 24.34441 & -45.74711 & 0.5 & 3.7 & 4.14 & 4.64 & 3.47 & 0.058 & 0.044 & 79.45 \\
\hline 79 & 24.34497 & -45.73313 & 0.6 & 2.86 & 4.06 & 5.07 & 2.99 & 0.065 & 0.038 & 77.98 \\
\hline 80 & 24.34648 & -45.71 & 0.5 & 1.48 & 4.2 & 4.66 & 3.5 & 0.057 & 0.042 & 82.48 \\
\hline 81 & 24.34841 & -45.73554 & 0.6 & 3 & 8.93 & 21.21 & 6.89 & 0.267 & 0.087 & 79.34 \\
\hline 82 & 24.34946 & -45.75306 & 0.8 & 4.05 & 4.23 & 6.66 & 4.14 & 0.085 & 0.053 & 78.34 \\
\hline 83 & 24.34988 & -45.71375 & 0.6 & 1.69 & 4.24 & 5.39 & 3.08 & 0.066 & 0.038 & 82.02 \\
\hline 84 & 24.35298 & -45.70252 & 0.5 & 1.02 & 4.69 & 5.61 & 4.59 & 0.068 & 0.056 & 82.67 \\
\hline 85 & 24.35363 & -45.74041 & 0.5 & 3.29 & 4.33 & 4.99 & 3.78 & 0.062 & 0.047 & 80.26 \\
\hline 86 & 24.35457 & -45.704 & 0.4 & 1.11 & 4.53 & 5.26 & 3.45 & 0.064 & 0.042 & 81.86 \\
\hline 87 & 24.35585 & -45.72007 & 0.6 & 2.08 & 4.07 & 5.23 & 3.05 & 0.067 & 0.039 & 77.78 \\
\hline 88 & 24.35658 & -45.74429 & 0.5 & 3.53 & 4.36 & 5.12 & 4.08 & 0.064 & 0.051 & 79.57 \\
\hline 89 & 24.35767 & -45.72204 & 0.5 & 2.2 & 4.12 & 4.62 & 3.61 & 0.058 & 0.046 & 79.37 \\
\hline 90 & 24.35804 & -45.71406 & 0.4 & 1.73 & 20.33 & 65.53 & 8.65 & 0.809 & 0.107 & 80.96 \\
\hline 91 & 24.36106 & -45.6992 & 0.4 & 0.92 & 8.1 & 15.9 & 5.78 & 0.198 & 0.072 & 80.52 \\
\hline 92 & 24.36306 & -45.76098 & 0.9 & 4.55 & 4 & 6.02 & 4.37 & 0.08 & 0.058 & 75.16 \\
\hline 93 & 24.36592 & -45.77341 & 1.8 & 5.31 & 4.61 & 13.47 & 5.57 & 0.198 & 0.082 & 68.1 \\
\hline 94 & 24.36836 & -45.74684 & 1.1 & 3.75 & 4.01 & 7.29 & 3.76 & 0.092 & 0.048 & 78.89 \\
\hline 95 & 24.36961 & -45.75132 & 1.2 & 4.02 & 6.78 & 18.65 & 6.54 & 0.244 & 0.086 & 76.3 \\
\hline 96 & 24.3703 & -45.74534 & 0.6 & 3.67 & 4.27 & 5.57 & 3.15 & 0.07 & 0.04 & 79.03 \\
\hline 97 & 24.37124 & -45.85096 & 2.6 & 9.96 & 4.01 & 14.78 & 7.44 & 0.381 & 0.192 & 38.78 \\
\hline 98 & 24.37154 & -45.71299 & 1 & 1.86 & 4.47 & 8.52 & 3.96 & 0.102 & 0.048 & 83.16 \\
\hline 99 & 24.3776 & -45.80918 & 2.5 & 7.5 & 4.61 & 17.64 & 6.77 & 0.288 & 0.11 & 61.24 \\
\hline 100 & 24.379 & -45.79162 & 2 & 6.47 & 4.52 & 13.67 & 6.32 & 0.203 & 0.094 & 67.41 \\
\hline 101 & 24.37945 & -45.71067 & 0.5 & 1.92 & 4.01 & 4.28 & 3.15 & 0.051 & 0.038 & 83.12 \\
\hline 102 & 24.3806 & -45.75672 & 0.9 & 4.44 & 4.17 & 6.06 & 5.3 & 0.089 & 0.078 & 68.21 \\
\hline 103 & 24.38111 & -45.7741 & 1.1 & 5.46 & 4.01 & 6.66 & 4.24 & 0.113 & 0.072 & 58.72 \\
\hline 104 & 24.38191 & -45.78949 & 1.1 & 6.37 & 18.99 & 76.61 & 10.38 & 1.131 & 0.153 & 67.77 \\
\hline 105 & 24.38656 & -45.71977 & 0.5 & 2.54 & 14.56 & 43.59 & 7.38 & 0.534 & 0.09 & 81.67 \\
\hline 106 & 24.38726 & -45.73216 & 0.4 & 3.18 & 4.32 & 4.87 & 3.27 & 0.062 & 0.042 & 78.16 \\
\hline 107 & 24.3882 & -45.71446 & 0.5 & 2.33 & 4.63 & 5.43 & 4.56 & 0.066 & 0.055 & 82.16 \\
\hline 108 & 24.38973 & -45.79828 & 3.3 & 6.95 & 4.17 & 20.68 & 7.29 & 0.322 & 0.114 & 64.15 \\
\hline 109 & 24.39143 & -45.83303 & 2.6 & 9.01 & 4.45 & 16.64 & 7.26 & 0.339 & 0.148 & 49.15 \\
\hline 110 & 24.39247 & -45.7863 & 1.6 & 6.29 & 4.05 & 9.12 & 5.11 & 0.134 & 0.075 & 67.81 \\
\hline 111 & 24.39273 & -45.78776 & 3.3 & 6.37 & 5.09 & 26.61 & 9.37 & 0.398 & 0.14 & 66.93 \\
\hline 112 & 24.39918 & -45.76846 & 1.1 & 5.37 & 4.1 & 7.4 & 4.49 & 0.104 & 0.063 & 71.27 \\
\hline 113 & 24.40015 & -45.71383 & 0.8 & 2.67 & 8.83 & 23.1 & 7.5 & 0.284 & 0.092 & 81.37 \\
\hline 114 & 24.40435 & -45.79253 & 2.6 & 6.79 & 4.05 & 15.04 & 6.15 & 0.233 & 0.095 & 64.48 \\
\hline 115 & 24.40516 & -45.75647 & 0.9 & 4.82 & 4.01 & 5.61 & 4.07 & 0.075 & 0.055 & 74.44 \\
\hline 116 & 24.40685 & -45.7565 & 1 & 4.86 & 4.94 & 9.23 & 4.34 & 0.124 & 0.059 & 74.17 \\
\hline 117 & 24.40963 & -45.75314 & 1.2 & 4.74 & 6.99 & 18.56 & 6.82 & 0.249 & 0.092 & 74.44 \\
\hline 118 & 24.40974 & -45.77543 & 1.4 & 5.93 & 4.31 & 9.88 & 4.57 & 0.146 & 0.068 & 67.47 \\
\hline 119 & 24.41799 & -45.7439 & 0.8 & 4.49 & 4.07 & 5.87 & 3.66 & 0.078 & 0.049 & 74.97 \\
\hline 120 & 24.41869 & -45.72115 & 0.5 & 3.55 & 4.02 & 4.14 & 2.98 & 0.059 & 0.043 & 69.93 \\
\hline 121 & 24.42167 & -45.73172 & 1 & 4.05 & 10.5 & 33.91 & 9.77 & 0.561 & 0.162 & 60.41 \\
\hline 122 & 24.42431 & -45.78277 & 1.4 & 6.59 & 4.62 & 10.94 & 6.05 & 0.179 & 0.099 & 61.11 \\
\hline 123 & 24.43091 & -45.73349 & 11.7 & 4.41 & 4.34 & 80.19 & 24.62 & 1.105 & 0.339 & 72.55 \\
\hline 124 & 24.44112 & -45.80277 & 1.6 & 7.98 & 6.57 & 21.88 & 7.92 & 0.564 & 0.204 & 38.81 \\
\hline 125 & 24.44278 & -45.77955 & 1.3 & 6.82 & 4.76 & 11.61 & 5.48 & 0.215 & 0.102 & 53.99 \\
\hline 126 & 24.46602 & -45.74354 & 1.1 & 5.94 & 4.28 & 7.94 & 4.59 & 0.135 & 0.078 & 58.79 \\
\hline 127 & 24.47931 & -45.77024 & 1.9 & 7.4 & 4.39 & 13.54 & 7.43 & 0.396 & 0.217 & 34.2 \\
\hline 128 & 24.50076 & -45.75685 & 2.8 & 7.59 & 4.4 & 3.27 & 3.24 & 0.253 & 0.251 & 12.93 \\
\hline 129 & 24.35544 & -45.66886 & 0.5 & 1.02 & 6.55 & 11.33 & 4.76 & 0.134 & 0.056 & 84.3 \\
\hline 130 & 24.36079 & -45.67592 & 0.5 & 0.71 & 4.13 & 4.97 & 3.73 & 0.059 & 0.044 & 84.13 \\
\hline 131 & 24.3612 & -45.67118 & 0.5 & 0.97 & 4.08 & 4.68 & 3.65 & 0.056 & 0.043 & 84.26 \\
\hline 132 & 24.36737 & -45.6892 & 0.5 & 0.72 & 4.84 & 6.47 & 5.45 & 0.107 & 0.09 & 60.29 \\
\hline 133 & 24.36739 & -45.66234 & 0.8 & 1.56 & 4.31 & 6.75 & 3.4 & 0.08 & 0.04 & 84.05 \\
\hline 134 & 24.37809 & -45.67449 & 0.5 & 1.32 & 4.23 & 5.13 & 4.29 & 0.061 & 0.051 & 83.65 \\
\hline 135 & 24.37872 & -45.64912 & 0.4 & 2.48 & 17.34 & 49.91 & 7.37 & 0.597 & 0.088 & 83.61 \\
\hline 136 & 24.37965 & -45.68606 & 2.4 & 1.2 & 4.52 & 20.53 & 7.68 & 0.247 & 0.092 & 83.14 \\
\hline 137 & 24.38679 & -45.6805 & 0.5 & 1.53 & 4.06 & 4.81 & 3.73 & 0.058 & 0.045 & 83.06 \\
\hline 138 & 24.38797 & -45.69146 & 0.7 & 1.59 & 5.78 & 11.13 & 4.65 & 0.142 & 0.059 & 78.64 \\
\hline
\end{tabular}


Table 2. continued.

\begin{tabular}{|c|c|c|c|c|c|c|c|c|c|c|}
\hline Num. & $\begin{array}{l}\text { RA (J2000) } \\
\text { deg }\end{array}$ & $\begin{array}{l}\text { Dec (J2000) } \\
\operatorname{deg}\end{array}$ & $\begin{array}{l}\text { Pos. err } \\
\text { arcsec }\end{array}$ & $\begin{array}{r}\text { Off-axis } \\
\text { arcmin }\end{array}$ & $\begin{array}{r}\text { Significance } \\
\sigma_{\mathrm{bkg}}\end{array}$ & Counts & Cts err. & \multicolumn{2}{|c|}{$\mathrm{ct} \mathrm{ks}^{-1}$} & $\begin{array}{l}\text { Exp. time } \\
\text { ks }\end{array}$ \\
\hline 139 & 24.39169 & -45.68939 & 0.5 & 1.72 & 11.44 & 33.53 & 9.75 & 0.427 & 0.124 & 78.53 \\
\hline 140 & 24.39241 & -45.6637 & 0.6 & 2.18 & 4.27 & 5.78 & 3.14 & 0.069 & 0.038 & 83.2 \\
\hline 141 & 24.39315 & -45.69359 & 0.5 & 1.83 & 4.41 & 5.34 & 4.42 & 0.065 & 0.054 & 82.36 \\
\hline 142 & 24.39479 & -45.67303 & 0.5 & 1.99 & 4.11 & 4.74 & 3.6 & 0.057 & 0.043 & 82.85 \\
\hline 143 & 24.39823 & -45.68351 & 0.5 & 1.99 & 4.42 & 5.39 & 4.39 & 0.065 & 0.053 & 82.43 \\
\hline 144 & 24.39822 & -45.66558 & 0.9 & 2.32 & 5.57 & 12.27 & 5.12 & 0.148 & 0.062 & 82.65 \\
\hline 145 & 24.40136 & -45.68145 & 0.4 & 2.13 & 17.74 & 54.75 & 8.16 & 0.666 & 0.099 & 82.25 \\
\hline 146 & 24.40198 & -45.64646 & 0.6 & 3.18 & 4 & 5.02 & 2.94 & 0.061 & 0.036 & 82 \\
\hline 147 & 24.40197 & -45.63096 & 0.8 & 3.92 & 4.44 & 6.82 & 3.41 & 0.084 & 0.042 & 81.12 \\
\hline 148 & 24.4051 & -45.69831 & 0.5 & 2.39 & 4.02 & 4.39 & 3.53 & 0.06 & 0.048 & 72.75 \\
\hline 149 & 24.4078 & -45.63746 & 0.5 & 3.75 & 4.14 & 4.51 & 3.49 & 0.056 & 0.043 & 81.1 \\
\hline 150 & 24.41299 & -45.70784 & 0.8 & 2.92 & 4.09 & 6.26 & 3.46 & 0.079 & 0.044 & 79.51 \\
\hline 151 & 24.41338 & -45.66728 & 0.5 & 2.84 & 4.27 & 4.84 & 4.04 & 0.059 & 0.05 & 81.48 \\
\hline 152 & 24.41502 & -45.70893 & 0.5 & 3.03 & 4.39 & 5.06 & 4.25 & 0.064 & 0.054 & 79.14 \\
\hline 153 & 24.41718 & -45.65529 & 0.5 & 3.32 & 4.24 & 4.72 & 4.05 & 0.062 & 0.054 & 75.68 \\
\hline 154 & 24.41836 & -45.61015 & 0.8 & 5.34 & 9.28 & 24.55 & 8.35 & 0.32 & 0.109 & 76.75 \\
\hline 155 & 24.42399 & -45.67821 & 0.8 & 3.09 & 7.75 & 19.93 & 6.67 & 0.284 & 0.095 & 70.2 \\
\hline 156 & 24.42602 & -45.66849 & 0.5 & 3.31 & 4.02 & 4.41 & 3.17 & 0.055 & 0.04 & 80.34 \\
\hline 157 & 24.42885 & -45.69185 & 0.6 & 3.29 & 4.05 & 5.3 & 3.07 & 0.067 & 0.039 & 79.36 \\
\hline 158 & 24.429 & -45.70179 & 0.5 & 3.41 & 4.58 & 5.52 & 4.42 & 0.073 & 0.058 & 75.9 \\
\hline 159 & 24.43106 & -45.60423 & 1.4 & 5.93 & 4.41 & 9.97 & 4.48 & 0.136 & 0.061 & 73.23 \\
\hline 160 & 24.43477 & -45.71769 & 0.8 & 4.01 & 4.6 & 7.35 & 3.58 & 0.097 & 0.047 & 75.56 \\
\hline 161 & 24.43754 & -45.71875 & 1 & 4.14 & 10.96 & 38.71 & 10.96 & 0.516 & 0.146 & 75.05 \\
\hline 162 & 24.43844 & -45.7195 & 0.8 & 4.19 & 4.49 & 7.18 & 3.84 & 0.096 & 0.051 & 74.78 \\
\hline 163 & 24.44013 & -45.65272 & 1 & 4.23 & 10.32 & 33.19 & 6.53 & 0.422 & 0.083 & 78.71 \\
\hline 164 & 24.44067 & -45.71887 & 1.1 & 4.26 & 4.06 & 7.64 & 4.09 & 0.103 & 0.055 & 74.49 \\
\hline 165 & 24.44059 & -45.61712 & 1.1 & 5.57 & 4.75 & 8.8 & 4.86 & 0.117 & 0.065 & 75.27 \\
\hline 166 & 24.44072 & -45.66278 & 1.3 & 4.01 & 5.43 & 14.27 & 5.67 & 0.193 & 0.077 & 73.93 \\
\hline 167 & 24.44157 & -45.65206 & 0.8 & 4.3 & 4.07 & 6.43 & 4.24 & 0.082 & 0.054 & 78.53 \\
\hline 168 & 24.4422 & -45.6679 & 2.5 & 3.97 & 4.29 & 17.66 & 6.99 & 0.228 & 0.09 & 77.44 \\
\hline 169 & 24.44296 & -45.69137 & 0.5 & 3.87 & 4.43 & 5.12 & 3.96 & 0.072 & 0.056 & 70.93 \\
\hline 170 & 24.44437 & -45.60236 & 1.4 & 6.35 & 4.44 & 10.3 & 4.84 & 0.147 & 0.069 & 70.05 \\
\hline 171 & 24.44484 & -45.70421 & 0.9 & 4.09 & 4.09 & 6.03 & 4.78 & 0.081 & 0.064 & 74.17 \\
\hline 172 & 24.46089 & -45.72968 & 3.6 & 5.31 & 4 & 21.36 & 8.32 & 0.32 & 0.125 & 66.65 \\
\hline 173 & 24.46353 & -45.69563 & 8.8 & 4.76 & 4.23 & 60.95 & 19.95 & 0.821 & 0.269 & 74.26 \\
\hline 174 & 24.46591 & -45.63691 & 1.2 & 5.64 & 6.91 & 19.22 & 6.85 & 0.274 & 0.098 & 70.04 \\
\hline 175 & 24.46607 & -45.69792 & 1 & 4.88 & 4.79 & 9.73 & 5.62 & 0.133 & 0.077 & 73.41 \\
\hline 176 & 24.46729 & -45.60975 & 1.4 & 6.67 & 4.73 & 11.3 & 4.93 & 0.169 & 0.074 & 66.81 \\
\hline 177 & 24.46935 & -45.64576 & 0.9 & 5.51 & 12.13 & 35.42 & 6.68 & 0.475 & 0.09 & 74.57 \\
\hline 178 & 24.47535 & -45.60512 & 1.9 & 7.11 & 4.39 & 13.23 & 5.73 & 0.208 & 0.09 & 63.65 \\
\hline 179 & 24.47657 & -45.71793 & 2.4 & 5.61 & 4.7 & 20.28 & 7.5 & 0.325 & 0.12 & 62.41 \\
\hline 180 & 24.48066 & -45.6784 & 1 & 5.45 & 4.34 & 8.65 & 5.18 & 0.118 & 0.071 & 73.19 \\
\hline 181 & 24.48415 & -45.64896 & 1.8 & 6 & 4.9 & 15.47 & 6.28 & 0.232 & 0.094 & 66.53 \\
\hline 182 & 24.4849 & -45.66845 & 1.2 & 5.71 & 13.99 & 58.3 & 8.68 & 0.801 & 0.119 & 72.77 \\
\hline 183 & 24.49416 & -45.6706 & 3.4 & 6.07 & 4.47 & 24.56 & 9.05 & 0.346 & 0.128 & 70.97 \\
\hline 184 & 24.49494 & -45.65133 & 0.9 & 6.38 & 11.68 & 38.63 & 7.04 & 0.567 & 0.103 & 68.12 \\
\hline 185 & 24.50131 & -45.69108 & 1.8 & 6.31 & 4.24 & 14.08 & 5.68 & 0.22 & 0.089 & 63.86 \\
\hline 186 & 24.50386 & -45.75107 & 3.4 & 7.51 & 4.04 & 23.21 & 8.35 & 0.951 & 0.342 & 24.42 \\
\hline 187 & 24.5116 & -45.701 & 2.4 & 6.8 & 4.67 & 19.63 & 7.38 & 0.357 & 0.134 & 54.91 \\
\hline 188 & 24.52064 & -45.64591 & 3.5 & 7.5 & 4.4 & 24.91 & 9.49 & 0.391 & 0.149 & 63.74 \\
\hline 189 & 24.52554 & -45.61767 & 3.6 & 8.38 & 4.07 & 20.84 & 8.19 & 0.392 & 0.154 & 53.19 \\
\hline 190 & 24.53792 & -45.65849 & 1.1 & 8.01 & 16.19 & 83.68 & 10.4 & 1.477 & 0.184 & 56.65 \\
\hline 191 & 24.59781 & -45.64849 & 4.3 & 10.59 & 5.62 & 42.6 & 13.48 & 1.433 & 0.454 & 29.72 \\
\hline 192 & 24.46743 & -45.9031 & 6.2 & 13.93 & 4.37 & 41.86 & 13.72 & 0.727 & 0.238 & 57.56 \\
\hline 193 & 24.48062 & -45.90942 & 8.6 & 14.48 & 4.36 & 58.98 & 18.88 & 0.921 & 0.295 & 64.05 \\
\hline 194 & 24.50558 & -45.84999 & 8.3 & 11.8 & 4.93 & 62.24 & 19.09 & 1.222 & 0.375 & 50.95 \\
\hline 195 & 24.51548 & -45.87582 & 8.1 & 13.33 & 9.98 & 218.01 & 36.58 & 3.389 & 0.569 & 64.33 \\
\hline 196 & 24.54034 & -45.86973 & 8 & 13.6 & 5.24 & 68.1 & 20.19 & 0.992 & 0.294 & 68.63 \\
\hline 197 & 24.55568 & -45.93656 & 11.4 & 17.32 & 4.58 & 95.27 & 28.35 & 1.514 & 0.451 & 62.9 \\
\hline 198 & 24.58917 & -45.93872 & 9.9 & 18.16 & 6.01 & 114.43 & 29.8 & 1.851 & 0.482 & 61.82 \\
\hline 199 & 24.60404 & -45.86263 & 11.3 & 15 & 4.91 & 86.87 & 25.58 & 1.257 & 0.37 & 69.08 \\
\hline 200 & 24.60963 & -45.91614 & 6.5 & 17.56 & 4.01 & 33.94 & 13.87 & 0.543 & 0.222 & 62.47 \\
\hline 201 & 24.62566 & -45.96008 & 12 & 20.08 & 4.32 & 75.66 & 23.79 & 2.25 & 0.708 & 33.62 \\
\hline 202 & 24.64624 & -45.87741 & 9.8 & 16.89 & 6.84 & 115.51 & 29.76 & 1.782 & 0.459 & 64.83 \\
\hline
\end{tabular}


Table 2. continued.

\begin{tabular}{|c|c|c|c|c|c|c|c|c|c|c|}
\hline Num. & $\begin{array}{l}\text { RA (J2000) } \\
\text { deg }\end{array}$ & $\begin{array}{l}\text { Dec (J2000) } \\
\text { deg }\end{array}$ & $\begin{array}{l}\text { Pos. err } \\
\text { arcsec }\end{array}$ & $\begin{array}{r}\text { Off-axis } \\
\text { arcmin }\end{array}$ & $\begin{array}{r}\text { Significance } \\
\sigma_{\mathrm{bkg}}\end{array}$ & Counts & Cts err. & \multicolumn{2}{|c|}{$\mathrm{ct} \mathrm{ks}^{-1}$} & $\begin{array}{r}\text { Exp. time } \\
\text { ks }\end{array}$ \\
\hline 203 & 24.54765 & -45.80043 & 4 & 10.74 & 4.64 & 61.5 & 18.26 & 0.957 & 0.284 & 64.28 \\
\hline 204 & 24.56448 & -45.78471 & 6.3 & 10.74 & 4.22 & 75.39 & 24.99 & 1.081 & 0.358 & 69.72 \\
\hline 205 & 24.63859 & -45.79607 & 6.9 & 13.75 & 12.84 & 583.33 & 73.27 & 5.914 & 0.743 & 98.63 \\
\hline 206 & 24.64888 & -45.7534 & 8.5 & 13.13 & 4.14 & 121.88 & 38.19 & 1.25 & 0.392 & 97.52 \\
\hline 207 & 24.65718 & -45.79576 & 6.2 & 14.43 & 4.1 & 87.83 & 28.55 & 0.9 & 0.293 & 97.54 \\
\hline 208 & 24.69243 & -45.86424 & 8.3 & 17.86 & 4.21 & 110.11 & 33.98 & 1.413 & 0.436 & 77.91 \\
\hline 209 & 24.6971 & -45.75497 & 6 & 15.09 & 4.36 & 94.03 & 29.94 & 0.971 & 0.309 & 96.81 \\
\hline 210 & 24.70579 & -45.71968 & 8.7 & 15.01 & 4.04 & 112.33 & 36.64 & 1.171 & 0.382 & 95.91 \\
\hline 211 & 24.74332 & -45.7744 & 5.9 & 17.28 & 18.76 & 917.15 & 82.52 & 10.289 & 0.926 & 89.14 \\
\hline 212 & 24.78107 & -45.75303 & 9.5 & 18.47 & 8.29 & 233.9 & 58.47 & 2.788 & 0.697 & 83.91 \\
\hline
\end{tabular}

